\title{
Pervasive RNA Regulation of Metabolism Enhances the Root Colonization Ability of Nitrogen-Fixing Symbiotic $\alpha$-Rhizobia
}

\author{
Natalia I. García-Tomsig, ${ }^{a}$ Marta Robledo, ${ }^{\text {a* }}$ George C. diCenzo, ${ }^{\text {b }}$ (D) Alessio Mengoni, ${ }^{c}$ Vicenta Millán, ${ }^{\text {a }}$ Alexandra Peregrina, ${ }^{\text {a }}$ \\ Alejandro Uceta, ${ }^{a}$ (D) José I. Jiménez-Zurdo ${ }^{a}$ \\ aStructure, Dynamics and Function of Rhizobacterial Genomes (Grupo de Ecología Genética de la Rizosfera), Estación Experimental del Zaidín, Consejo Superior de \\ Investigaciones Científicas (CSIC), Granada, Spain \\ bDepartment of Biology, Queen's University, Kingston, Ontario, Canada \\ cDepartment of Biology, University of Florence, Sesto Fiorentino, Italy
}

ABSTRACT The rhizosphere and rhizoplane are nutrient-rich but selective environments for the root microbiome. Here, we deciphered a posttranscriptional network regulated by the homologous trans-small RNAs (sRNAs) AbcR1 and AbcR2, which rewire the metabolism of the nitrogen-fixing $\alpha$-rhizobium Sinorhizobium meliloti during preinfection stages of symbiosis with its legume host alfalfa. The LysR-type regulator LsrB, which transduces the cell redox state, is indispensable for AbcR1 expression in actively dividing bacteria, whereas the stress-induced transcription of AbcR2 depends on the alternative $\sigma$ factor RpoH1. MS2 affinity purification coupled with RNA sequencing unveiled exceptionally large and overlapping AbcR1/2 mRNA interactomes, jointly representing $\sim 6 \%$ of the $S$. meliloti protein-coding genes. Most mRNAs encode transport/metabolic proteins whose translation is silenced by base pairing to two distinct anti-Shine Dalgarno motifs that function independently in both sRNAs. A metabolic model-aided analysis of the targetomes predicted changes in AbcR1/2 expression driven by shifts in carbon/nitrogen sources, which were confirmed experimentally. Low AbcR1/2 levels in some defined media anticipated overexpression growth phenotypes linked to the silencing of specific mRNAs. As a proof of principle, we confirmed AbcR1/2-mediated downregulation of the L-amino acid AapQ permease. AbcR1/2 interactomes are well represented in rhizosphere-related $S$. meliloti transcriptomic signatures. Remarkably, a lack of AbcR1 specifically compromised the ability of $S$. meliloti to colonize the root rhizoplane. The AbcR1 regulon likely ranks the utilization of available substrates to optimize metabolism, thus conferring on S. meliloti an advantage for efficient rhizosphere/rhizoplane colonization. AbcR1 regulation is predicted to be conserved in related $\alpha$-rhizobia, which opens unprecedented possibilities for engineering highly competitive biofertilizers.

IMPORTANCE Nitrogen-fixing root nodule symbioses between rhizobia and legume plants provide more than half of the combined nitrogen incorporated annually into terrestrial ecosystems, rendering plant growth independent of environmentally unfriendly chemical fertilizers. The success of symbiosis depends primarily on the capacity of rhizobia to establish competitive populations in soil and rhizosphere environments. Here, we provide insights into the regulation and architecture of an extensive RNA posttranscriptional network that fine-tunes the metabolism of the alfalfa symbiont $S$. meliloti, thereby enhancing the ability of this beneficial bacterium to colonize nutrient-rich but extremely selective niches, such as the rhizosphere of its host plant. This pervasive RNA regulation of metabolism is a major adaptive mechanism, predicted to operate in diverse rhizobial species. Because RNA regulation relies on modifiable base-pairing interactions, our findings open unexplored avenues for engineering the legumes rhizobiome within sustainable agricultural practices.
Invited Editor Masatoshi Miyakoshi, University of Tsukuba

Editor Joerg Vogel, University of Würzburg

Copyright $\odot 2022$ García-Tomsig et al. This is an open-access article distributed under the terms of the Creative Commons Attribution 4.0 International license.

Address correspondence to José I. JiménezZurdo, jijz@eez.csic.es.

*Present address: Marta Robledo, Intergenomics Group, Departamento de Biología Molecular, Instituto de Biomedicina y Biotecnología de Cantabria, CSIC-Universidad de Cantabria-Sodercan, Santander, Spain.

§Present address: Alexandra Peregrina, Control of Gene Expression Lab, Instituto de Tecnologia Química e Biológica António Xavier, Universidade Nova de Lisboa, Oeiras, Portugal.

The authors declare no conflict of interest.

Received 26 November 2021

Accepted 26 January 2022

Published 15 February 2022 
KEYWORDS Sinorhizobium meliloti, alphaproteobacteria, noncoding RNA, riboregulation

itrogen-fixing root nodule symbioses established between some soil-dwelling alpha- or betaproteobacteria, known as rhizobia, and legume plants are pillars of agro-environmental sustainability (1). These mutualistic species-specific plant-microbe interactions rely on a strict metabolic cooperation between the partners. Rhizobia must cope with drastic shifts in nutrient availability during colonization of soil, rhizosphere, and root cells $(2,3)$. Bulk soil is a heterogeneous oligotrophic environment commonly exposed to abiotic stress. Plant-derived nutrients fuel the metabolism of rhizospheric and invading rhizobia, as well as symbiotic nitrogen fixation by morphologically differentiated bacteroids within nodules. Thus, the success of a symbiosis greatly depends on the ability of rhizobia to accurately adapt their metabolism to the nutritionally complex soil and plant environments. The metabolic versatility of rhizobia is supported by large and multipartite genomes, with a generous genetic endowment arranged in complex networks devoted to nutrient uptake and catabolism $(4,5)$. To date, regulation of these metabolic pathways has been almost exclusively attributed to proteins involved in differential transcription (i.e., transcription factors and alternative RNA polymerase holoenzymes) or specific posttranslational modifications, but the underlying posttranscriptional mechanisms are largely unknown (6-9). However, since the massive discovery of small noncoding RNAs (sRNAs) in prokaryotes, RNA-mediated posttranscriptional control of metabolism has been regarded as a ubiquitous level of regulation, contributing greatly to bacterial fitness in fluctuating environments (10).

The best-characterized noncoding transcriptome in rhizobia is that of the alphaproteobacterium Sinorhizobium meliloti, the symbiont of alfalfa (Medicago sativa L.) and other medic legumes (11-13). Transcriptome sequencing (RNA-seq) surveys have unveiled a large and heterogeneous population of noncoding RNAs expressed by $S$. meliloti, but only a few so-called trans-sRNAs involved in regulating quorum sensing, the cell cycle, and metabolism have been further characterized (14-20). trans-acting sRNAs constitute the most studied class of riboregulators in bacteria. It includes transcripts mostly encoded within intergenic regions (IGRs) that typically influence the translation and/or half-life of multiple trans-encoded mRNAs upon protein-assisted short and imperfect base pairing (21). Computational predictions and preliminary experimental data suggest that at least three of the characterized S. meliloti trans-sRNAs, namely, NfeR1, AbcR1, and $A b c R 2$, regulate nutrient uptake by inhibiting the translation of mRNAs encoding components of $A B C$ transporters $(14,15,22)$.

NfeR1 (nodule formation efficiency RNA 1) is a 115-nucleotide (nt) stress-induced sRNA required for osmoadaptation and nodule development (14). The regulatory ability of NfeR1 depends on three identical anti-Shine-Dalgarno (aSD) motifs that contribute redundantly to translation inhibition. Only two mRNAs have been experimentally validated as NfeR1 targets, SMc03121 and SMb20442, both of which encode periplasmic substrate-binding proteins of yet-uncharacterized transporters. AbcR1 and AbcR2 (ATP-binding cassette regulators 1 and 2) are 121-nt- and 114-nt-long homologous sRNAs tandemly carried in the IGR flanked by the genes SMc01226 and IsrB, which encode an ArsR-type and the LsrB (LysR-type symbiotic regulator B) transcriptional regulators, respectively (15). Unlike NfeR1, AbcR1/2 interact with Hfq, the widespread bacterial chaperone that commonly acts as an RNA matchmaker in riboregulation (22). Despite their homology, these sRNAs are differentially expressed (15). AbcR1 expression is induced during exponential growth and at early root infection, while it is downregulated in nitrogen-fixing endosymbiotic bacteroids. Conversely, the highest levels of AbcR2 accumulation occur at the onset of stationary-phase growth and in response to abiotic stress, whereas it is barely detected in planta throughout symbiosis. Accordingly, only a lack of AbcR1 results in a growth delay in rich medium, and both AbcR1 and AbcR2 are dispensable for wild-type nodule organogenesis and nitrogen fixation in symbiosis with $M$. sativa plants (15). 
S. meliloti AbcR1/2 are founding members of the so-called $\alpha \mathrm{r} 15$ family of alphaproteobacterial sRNAs, which has representatives in most Rhizobiaceae and Brucellaceae species, including the mammal and plant pathogens Brucella abortus and Agrobacterium tumefaciens (23-25). Remarkably, AbcR1 and AbcR2 are functionally redundant for $B$. abortus virulence; i.e., only a double deletion prevents chronic infection of host macrophages $(26,27)$. In $A$. tumefaciens and $B$. abortus, AbcR1 expression is regulated by $\mathrm{VtIR}$, the LysR-type transcriptional regulator (LTTR) encoded by the neighboring gene $(28,29)$. The AbcR1 genomic region shows high synteny across alphaproteobacteria, and therefore, the S. meliloti ortholog LsrB has been proposed as the most probable AbcR1 regulator in this bacterium. However, this hypothesis has not yet been experimentally demonstrated (30). Transcriptomic and proteomic signatures of knockout mutants have drafted the regulons of $B$. abortus and A. tumefaciens $\operatorname{AbcR1/2}(26,27,31)$. Although such profiling does not discriminate between directly and indirectly regulated mRNAs, differentially expressed transcripts were functionally enriched in nutrient uptake and virulence factors. Further experimental validation demonstrated that subsets of these target mRNA candidates are regulated through either of the two aSD motifs that are identifiable as singlestranded regions in the predicted secondary structures of $\alpha \mathrm{r} 15$ sRNAs $(27,31)$. Similarly, genetic reporter assays have confirmed livK, prbA, and SMa0495, all encoding the periplasmic component of $A B C$ transporters for amino acid uptake, as targets of $S$. meliloti AbcR1/2 $(15,22)$. These mRNAs are most probably regulated by base pairing of the aSD motifs to the ribosome binding site (RBS) and flanking nucleotides, but these interactions have not yet been genetically dissected.

Here, we explored the regulation of the $S$. meliloti AbcR1/2 sRNAs and uncovered their mRNA interactomes using MAPS (MS2 affinity purification coupled with RNA sequencing) $(32,33)$. Our data show that LsrB and the alternative $\sigma$ factor RpoH1 are responsible for the differential transcription of $A b c R 1$ and $A b c R 2$, respectively. In turn, these sRNAs use their aSD motifs to downregulate the translation of large and overlapping arrays of mRNAs encoding transport proteins and metabolic enzymes. Further, we show that AbcR1-mediated posttranscriptional fine-tuning of metabolism enhances the ability of $S$. meliloti to colonize the root rhizoplane, a biotechnologically relevant symbiotic trait.

\section{RESULTS}

Regulators of AbcR1/2 transcription. To identify putative functional motifs in the AbcR1/2 promoters $\left(P_{a b c R 1}\right.$ and $P_{a b c R 2}$ ), we compared the 100 nucleotides preceding the transcription start sites of these genes and their predicted homologs in several alphaproteobacteria (see Fig. S1A in the supplemental material). $\mathrm{P}_{a b c R 1}$ alignment unveiled the $-35 /-10$ core hexamers recognized by $\operatorname{RpoD}\left(\sigma^{70}\right)$ in alphaproteobacteria (CTTGAC$\mathrm{N}_{17}$-CTATAT) (11). Upstream of this $\sigma^{70}$ signature, we noticed the generic LTTR motif of prokaryotic promoters ( $\left.\mathrm{T}-\mathrm{N}_{11}-\mathrm{A}\right)$, which occurs in tandem in most sequences. Remarkably, a more defined motif perfectly matching the proposed LsrB-binding consensus GCAT- $\mathrm{N}_{3}-\mathrm{TG}-\mathrm{N}_{3}-\mathrm{T}$ in $B$. abortus and $A$. tumefaciens was also evident between the -61 and -49 positions in $\mathrm{P}_{a b c R 1}$. Comparison of the $\mathrm{P}_{a b c R 2}$ sequences revealed a -35 / -10 box closely matching the $S$. meliloti RpoH1 $\left(\sigma^{\mathrm{H} 1}\right)$ consensus sequence (CTTGAA$\mathrm{N}_{16}$-CCTATAT) but failed to detect additional conserved motifs (34).

To experimentally test these predictions, we first transcriptionally fused full-length $\left(\mathrm{P}_{a b c R 1} / \mathrm{P}_{a b c R 2}\right)$ and truncated $\left(\mathrm{P}_{a b c R 1-38} / \mathrm{P}_{a b c R 2-38}\right)$ versions of both promoters to the enhanced green fluorescent protein gene (eGFP). All four reporter constructs were independently introduced into wild-type S. meliloti (Sm2011 and Sm1021) and IsrB $(\mathrm{Sm} \Delta / \mathrm{srB}), r p o H 1$, and $r p o H 2$ knockout mutant strains (Fig. 1A). Strains Sm2011 and Sm1021 (reference genome) derive from the same S. meliloti nodule isolate (SU47). Both are considered nearly isogenic (Table S1), but strikingly, we failed to generate an IsrB knockout in Sm1021 using both single- and double-crossover strategies, suggesting that the genomic background can affect IsrB mutant viability. The activities of the promoters were assessed in bacteria grown to exponential $\left(\mathrm{P}_{a b c R 1}\right)$ or stationary $\left(\mathrm{P}_{a b c R 2}\right)$ 


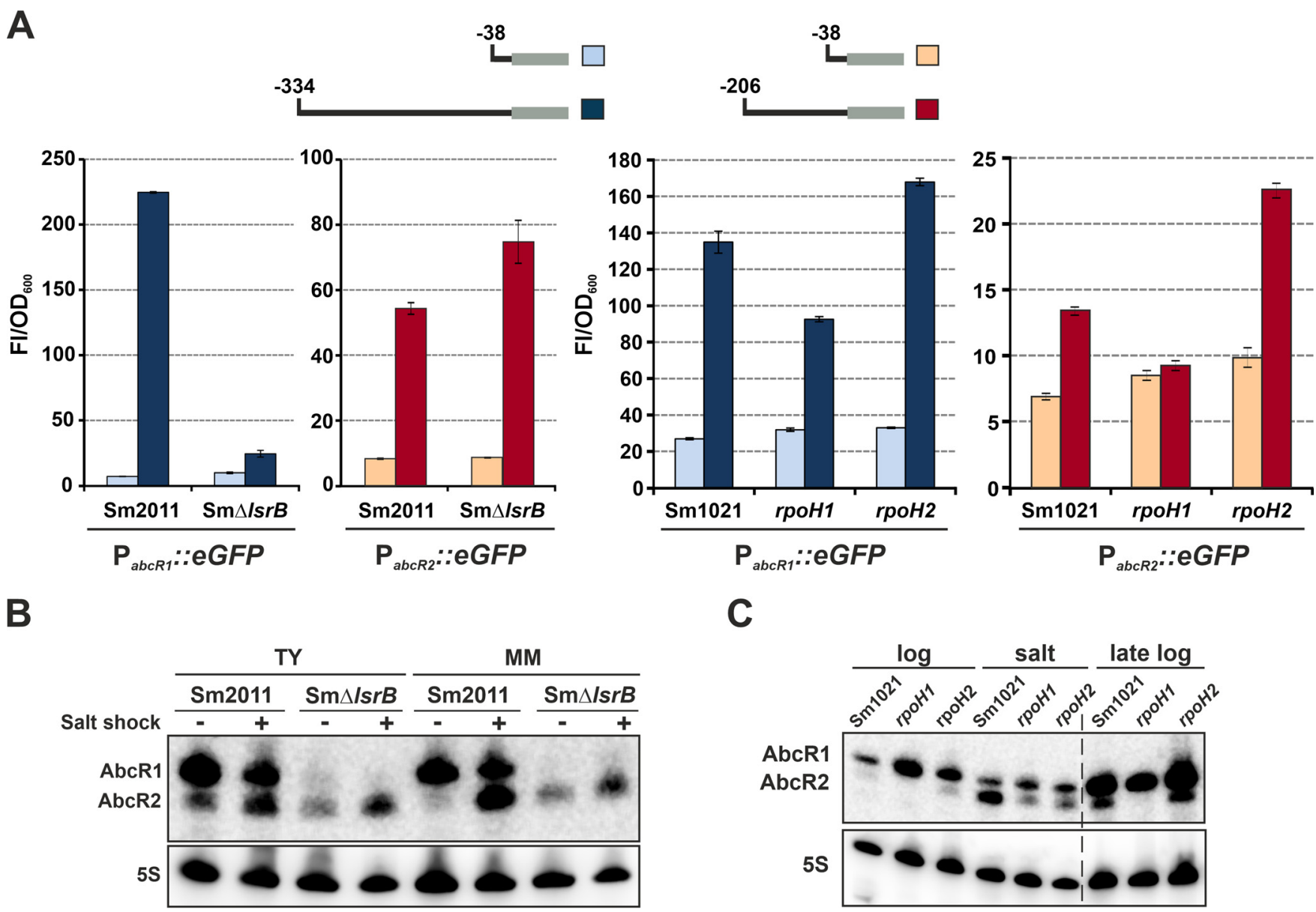

FIG 1 Transcriptional regulation of S. meliloti AbcR1 and AbcR2 sRNAs. (A) Fluorescence of promoter-eGFP fusions. Fluorescence derived from full-length and trimmed versions of $P_{a b c R 1}$ and $P_{a b c R 2}$, as diagrammed above the bar graphs, were determined in wild-type (Sm2011 or Sm1021) and mutant IsrB $(\mathrm{Sm} \Delta / \mathrm{srB}), r p o H 1$, and $r p o H 2$ backgrounds. Reported values are means and standard deviations (SD) of nine fluorescence measurements normalized to the $\mathrm{OD}_{600}\left(\mathrm{FI} / \mathrm{OD}_{600}\right)$ of exponential $\left(\mathrm{P}_{a b c \mathrm{R} 1}\right)$ - and stationary $\left(\mathrm{P}_{a b c \mathrm{C} 2}\right)$-phase cultures, i.e., three replicates of three independent cultures of each reporter strain. $(\mathrm{B})$ Northern blot analysis of LsrB-dependent AbcR1/2 expression. Total RNA was obtained from Sm2011 and Sm $\Delta /$ srB bacteria. (C) Northern blot analysis of $\mathrm{RpoH} 1 / 2$-dependent AbcR1/2 expression. Total RNA was extracted from Sm1021 and rpoH1 rpoH2 knockout mutants. Growth conditions are indicated on top of each panel. Membranes were probed with the PbAbcR1/2-radiolabeled oligonucleotide. The 5S rRNA was probed as an RNA loading control. Hybridizations shown are representatives of at least three biological replicates per strain and growth condition.

phase in complete tryptone-yeast (TY) medium, which induces AbcR1 or AbcR2 expression, respectively. The maximum fluorescence of the $P_{a b c k i}:: e G F P$ reporter fusion was detected in Sm2011, decreasing by $>22$-fold upon removal of the predicted LsrB-binding motif. In the absence of $L s r B$, the activity of $\mathrm{P}_{a b c R 1}$ was merely double that of $\mathrm{P}_{a b c R 1-38}$. Conversely, the lack of LsrB did not significantly alter $P_{a b c k 2}$ 's activity. Gel shift assays further demonstrated the binding of $\mathrm{LsrB}$ to $\mathrm{P}_{a b c k 1}$ (Fig. S1B). In strain Sm1021 and its $r p o H$ insertion mutants (34), $\mathrm{P}_{a b c R 1}$-derived fluorescence was 3- to 6-fold higher than that derived from $\mathrm{P}_{a b c R 1-38}$. In contrast, transcription from $\mathrm{P}_{a b c k 2}$ specifically decreased in the rpoH1 mutant to the basal levels rendered by $\mathrm{P}_{a b c R 2-38}$. The strongly reduced activity of $\mathrm{P}_{a b c k 2-38}$, relative to that of $\mathrm{P}_{a b c R 2}$ in the wild-type and mutant backgrounds is likely due to a deletion of transcriptional enhancers located upstream of many RpoH boxes, which remain uncharacterized in S. meliloti (34).

In an independent series of experiments, we used the same set of S. meliloti strains to examine AbcR1 and AbcR2 accumulation in different media and growth conditions by Northern blotting probing of total RNA with an oligonucleotide that cross-reacts with both transcripts (Fig. 1B and C). Hybridization of RNA from Sm2011 confirmed high levels of AbcR1 during exponential growth and an increased abundance of AbcR2 upon an osmotic upshift in both complete TY medium and defined minimal medium (MM) (Fig. 1B). In contrast, whereas AbcR2 retained its stress-induced expression in 
$\mathrm{Sm} \Delta / s r B, A b c R 1$ was undetectable in this mutant. In S. meliloti, RpoH1 and RpoH2 are coproduced at the onset of stationary-phase growth in MM and in response to a salt shock (34). Thus, we used these conditions to further assess RpoH-dependent expression of $A b c R 1 / 2$ by Northern hybridization (Fig. 1C). Probing of RNA from strain Sm1021 confirmed AbcR2 expression during stationary phase and under salt stress. Unlike AbcR1, which accumulated in the absence of either $\sigma^{\mathrm{H}}$ factor, AbcR2 was not present in unstressed bacteria lacking $\mathrm{RpoH} 1$ and was downregulated, but reliably detected, in both $\mathrm{rpoH}$ mutants upon a salt shock. Therefore, it is likely that $\mathrm{RpoH} 1$ and $\mathrm{RpoH} 2$ contribute additively to AbcR2 transcription under this specific condition. This is not surprising given the similarities between the $\sigma^{\mathrm{H} 1}$ and $\sigma^{\mathrm{H} 2}$ motifs in S. meliloti promoters (34). To confirm this observation, we probed new RNA samples with an oligonucleotide specifically targeting AbcR2 (Fig. S1C). In this experiment, we also included the $\mathrm{rpoH} 1 \mathrm{rpoH} 2$ double-insertion mutant, as well as RNA from bacteria subjected to a heat shock $\left(40^{\circ} \mathrm{C}\right)$, which also promotes the activities of both $\sigma^{\mathrm{H}}$ factors. The lack of $\mathrm{RpoH} 1$ was enough to render AbcR2 undetectable under all conditions except salt stress, under which complete inhibition of AbcR2 expression required the double knockout. Together, these data revealed that the transcriptional regulation of $A b c R 1$ and AbcR2 mostly depends on LsrB and RpoH1, respectively.

MAPS-based characterization of the AbcR1/2 targetomes. We used MAPS to identify the AbcR1/2 mRNA partners at a genome-wide scale. For this, we fused the MS2 RNA aptamer to the $5^{\prime}$ ends of AbcR1/2, which allows for the specific capture of the tagged transcripts along with their interacting mRNAs by an MS2-MBP (maltose-binding protein) fusion protein immobilized on an amylose resin. Tagging at the $5^{\prime}$ end was previously shown to preserve the stable expression and functional secondary structure of the AbcR sRNAs $(35,36)$. Given that AbcR1/2 and NfeR1 are predicted to share targets (14), wild-type AbcR1/2 (controls) and tagged AbcR1/2 were expressed from an isopropyl- $\beta$-D-thiogalactopyranoside (IPTG)-inducible promoter in the $\Delta a b c R 1$ $\Delta a b c R 2 \Delta n f e R 1$ strain Sm2020 (an Sm2011 derivative). The sRNAs were reliably detected as transcripts of the expected sizes in total RNA extractions following 15, 30, and 60 min of IPTG addition (Fig. S2A). The chimeric transcripts also retained the ability to downregulate $p r b A$, as shown using a prbA::eGFP translational fusion (Fig. S2B). These data validated the tagged sRNAs as baits for affinity chromatography.

To maximize copurification of target mRNAs and targetome coverage, we induced the transcription of wild-type controls and MS2-sRNAs for a short time (15 min) under conditions that promote their endogenous expression, i.e., exponential growth in TY and MM for $A b c R 1$, and stationary-phase growth (TY and MM) as well as heat and salt shocks (both in TY) for AbcR2. Lysates from pools of AbcR1 and AbcR2 cultures were then subjected to affinity chromatography. Hybridization of RNA from the input and output chromatography fractions showed that the baits were specifically retained by the MS2-MBP protein (Fig. S2C). Mapping of the sequencing reads from the eluted RNA to the S. meliloti reference genome (Sm1021) unequivocally demonstrated efficient recovery of the tagged sRNAs and copurification of the known prbA and SMa0495 targets (22), as expected (Fig. S2C and D).

Upon normalization by coverage, we compared read counts derived from controls and MS2-AbcR1/2 mapping to those of four mRNA regions: (i) the full-length mRNA, including the coding sequence and a virtual $5^{\prime}$ untranscribed region (5'-UTR) of $50 \mathrm{nt}$, (ii) a stretch of the $5^{\prime}$ region extending from nucleotide positions -50 to +100 relative to the translation start codon, (iii) the coding sequence alone, and (iv) the $3^{\prime}$ region encompassing $50 \mathrm{nt}$ upstream and $30 \mathrm{nt}$ downstream of the stop codon as a virtual $3^{\prime}$ UTR. We imposed a minimum of 50 (MS2-AbcR1) or 25 (MS2-AbcR2) mapped sequencing reads for an mRNA region to be considered in the comparisons. An mRNA was scored as a putative AbcR1/2 target if counts from tagged sRNA libraries exceeded a 3fold difference ( $\log _{2}$ fold change $\left[\log _{2} F C\right]>1.5$ ) from counts for the controls in at least one of the computed regions. IntaRNA-predicted antisense interactions using the tagged sRNAs as queries were then used to filter out those mRNAs likely captured by unspecific binding to the MS2 aptamer. All three previously identified AbcR1/2 target 


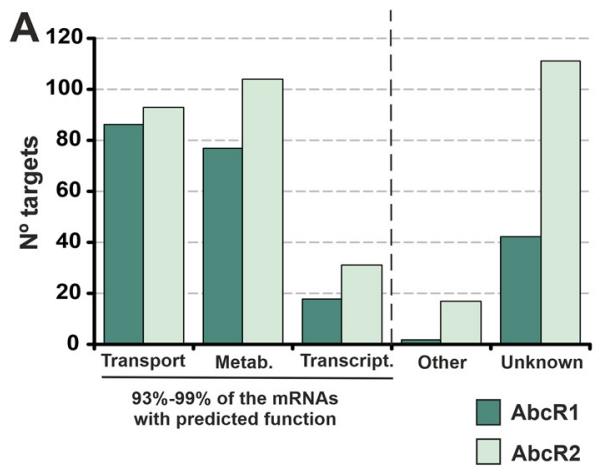

B

mRNA targets uncovered by MAPS
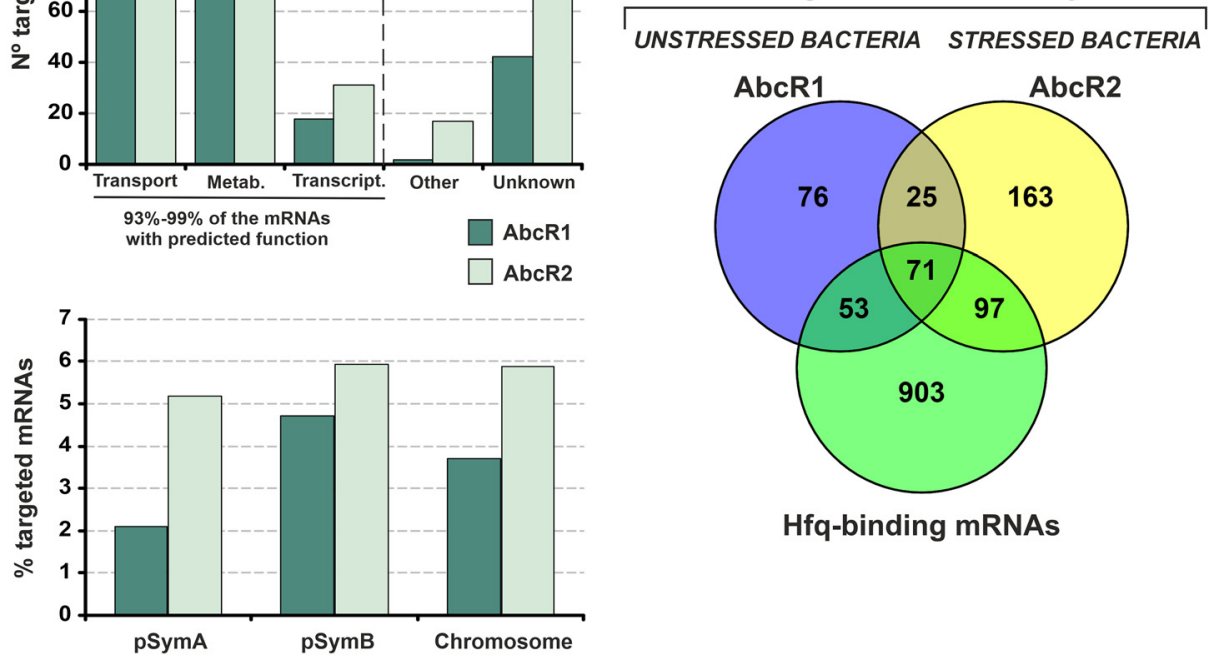

Hfq-binding mRNAs

FIG 2 Overview of the AbcR1/2 mRNA interactomes determined by MAPS. (A) Functional categories of the captured mRNAs (top) and their distribution relative to the total number of protein-coding genes in each of the three S. meliloti replicons: the pSymA and pSymB megaplasmids and the chromosome (bottom). Metab., metabolism mRNAs; Transcript., transcription mRNAs. (B) Venn diagram comparing the AbcR1/2 targetomes and the set of known Hfq-binding mRNAs.

mRNAs (livK, prbA, and SMa0495) passed the selection thresholds. All in all, MAPS identified 225 and 356 interacting mRNAs for AbcR1 and AbcR2, respectively, representing roughly $4 \%$ to $6 \%$ of $S$. meliloti protein-coding genes (Data Set S1).

AbcR1/2 broadly regulate S. meliloti metabolism. According to the Sm1021 genomic sequence annotation (37), $70 \%$ to $80 \%$ of the AbcR1/2 target mRNA candidates encode proteins with predicted functions (Fig. 2A). Of those, 72\% (AbcR1) and 55\% (AbcR2) are most probably involved in the transport or metabolism of widely diverse substrates. An additional $8 \%$ to $9 \%$ of both targetomes encode transcription factors, many of which are linked to metabolic operons. Collectively, the sets of metabolism-associated mRNAs represent $93 \%$ to $99 \%$ of the AbcR1 and AbcR2 targets with functional homology in database entries. The relative distribution of both targetomes along the three $S$. meliloti replicons indicates that the impact of $A b c R 1 / 2$-mediated posttranscriptional regulation is slightly biased toward the pSymB megaplasmid (Fig. 2A), which is enriched in metabolic genes. Furthermore, pangenome analysis of 23 complete S. meliloti genomes identified 153 (68\% of the total) and 215 (60.4\%) AbcR1 and AbcR2 targets, respectively, as belonging to the core genome (Data Set S1). Although the different experimental setups (i.e., growth conditions) preclude a rigorous comparison between the AbcR1 and AbcR2 targetomes, MAPS uncovered 96 common targets for both sRNAs (Fig. 2B). Interestingly, 221 (20\%) of the 1,127 mRNAs previously identified as Hfq ligands were also scored as AbcR1/2 targets (22), which indicates a prominent role of $A b c R 1 / 2$ in the regulation of the extensive $S$. meliloti Hfq posttranscriptional network. The partial overlap between the AbcR1/2 targetomes and the Hfq partners suggests that most of these mRNAs are true sRNA targets rather than transcripts recovered unspecifically solely by binding to $\mathrm{Hfq}$. This analysis thus anticipates a pervasive AbcR1/2 regulation of $S$. meliloti's adaptive metabolism.

AbcR1/2 use two distinct aSD motifs for regulation by base pairing. Enrichmentbased clustering of the mRNAs copurified with tagged AbcR1/2 unveiled three groups of targets, which were characterized by sequencing coverage biases toward either the $5^{\prime}$ region (cluster I), the coding sequence (cluster II), or the $3^{\prime}$ region (cluster III) (Fig. 3 ). Cluster I was the dominant in both targetomes. IntaRNA predictions revealed a correlation between the enrichment of a specific mRNA region and the location of the expected 


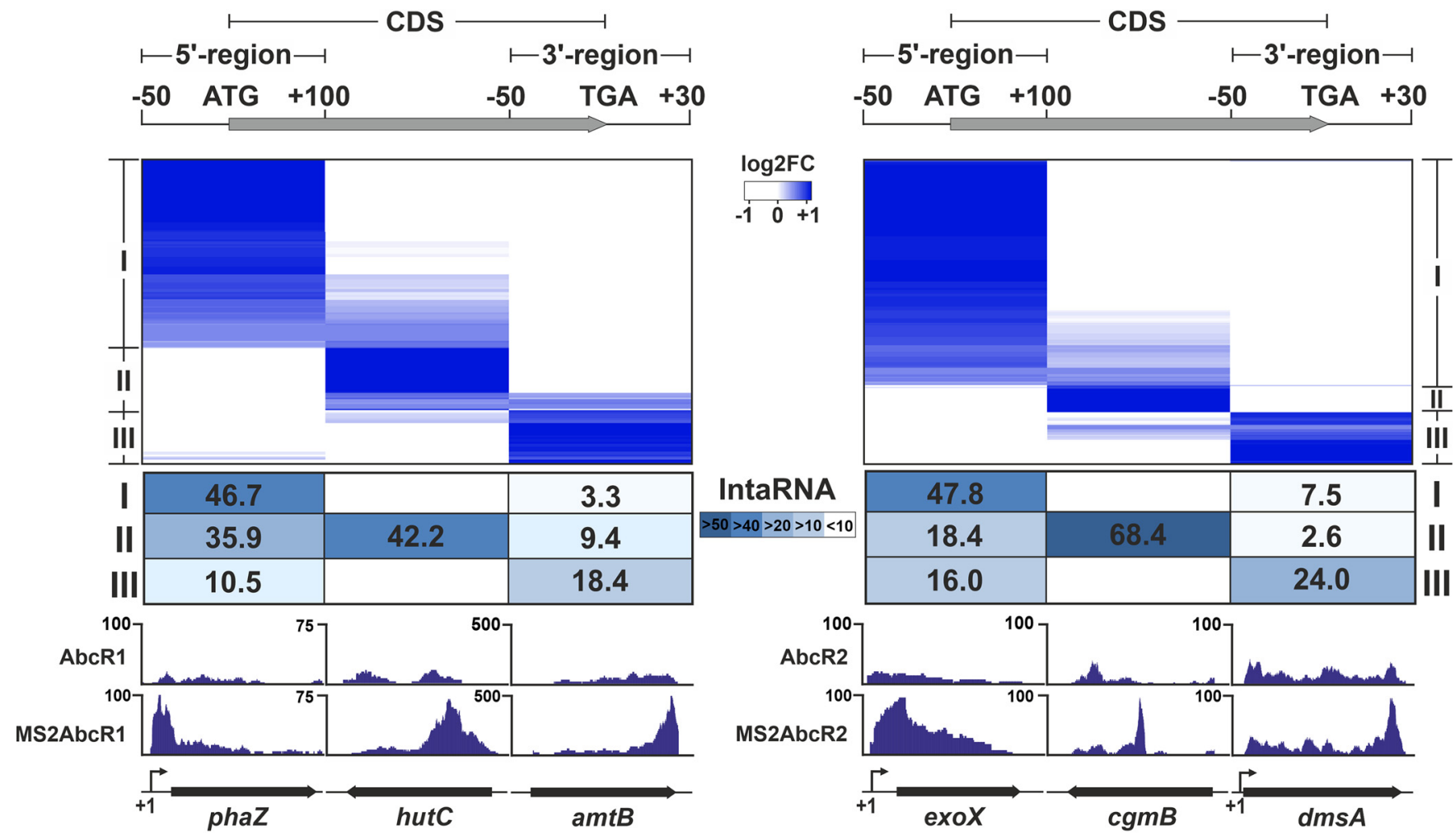

FIG 3 Enrichment-based clustering of target mRNAs upon affinity chromatography with MS2-AbcR1/2. Data for AbcR1 and AbcR2 are shown on the left and the right, respectively. Heatmaps identify three groups of mRNAs enriched at the $5^{\prime}$ region (cluster I), coding sequence (CDS; cluster II), and $3^{\prime}$ region (cluster III) with respect to the control experiments with the wild-type sRNAs ( $\log _{2} \mathrm{FC}$ scale); these three regions are shown schematically at the top of the figure. The S. meliloti genome was interrogated with IntaRNA for thermodynamically favored $(E<-8)$ antisense interactions (minimum, 7-nt seed) of $A b c R 1 / 2$ in each of the mRNA regions. Numbers in the table indicate the percentage of mRNAs in each cluster that have a predicted antisense interaction within each region and is presented as a heatmap (legend between tables). Interactions predicted in the CDS may overlap those at the $5^{\prime}$ or $3^{\prime}$ mRNA region, and therefore, numbers in columns and rows may add up to more than $100 \%$. IGV plots at the bottom show read coverages of target mRNAs representatives of each cluster.

antisense AbcR1/2 interaction sites (Fig. 3). The target mRNAs livK, prbA, and SMa0495 are representatives of the dominant cluster, cluster I. They were previously validated by means of translational fusions of their 5 ' regions to eGFP as reporters of AbcR1/2-dependent regulation $(15,22)$. Here, we used a similar genetic reporter assay to validate a new set of three target mRNA candidates within cluster I that encode transport proteins: SMc02417, SMc03121, and SMa0392 (Fig. S3A). IPTG-induced (over)expression of AbcR1/2 reduced fluorescence from the three reporters significantly, indicating downregulation of translation and equivalent regulatory abilities of the two sRNAs.

The overlapping targeting potential of AbcR1/2 likely relies on identical pairs of 8-nt aSD motifs located at the loop of the $5^{\prime}$ hairpin (CUCCUCCC; aSD1) and between SL1 and SL2 stem loops (UUCCCCUC; aSD2) in both molecules (Fig. 4A). To pinpoint the contribution of aSD1 and aSD2 to regulation, we first assessed the activities of two AbcR1/2 variants on the SMc03121 and SMa0495 targets using the translational reporter fusion assay. Specifically, we introduced 2-nucleotide substitutions within either aSD1 (AbcR1a/AbcR2a) or aSD2 (AbcR1b/AbcR2b) that preserve the putative secondary structures of both transcripts while disrupting the predicted base pairing at the translation initiation region of the target mRNAs (Fig. 4A and B). Interaction with SMc03121 probably occurs via aSD1, whereas SMa0495 is likely targeted by aSD2 (Fig. 4B). Induced (over)expression of wildtype $A b c R 1 / 2$ resulted in a decrease of reporter-derived fluorescence in both cases. Consistently with the predicted interactions, AbcR1/2a variants (aSD1 mutants) retained wild-type activity on SMa0495 but lost the ability to repress SMc03121, whereas the regulatory effects were the opposite with mutants in aSD2 (Fig. 4C). SMc03121 is thus a common target of NfeR 1 and AbcR1/2, which is regulated by interactions of aSD seeds at the RBS. 
A
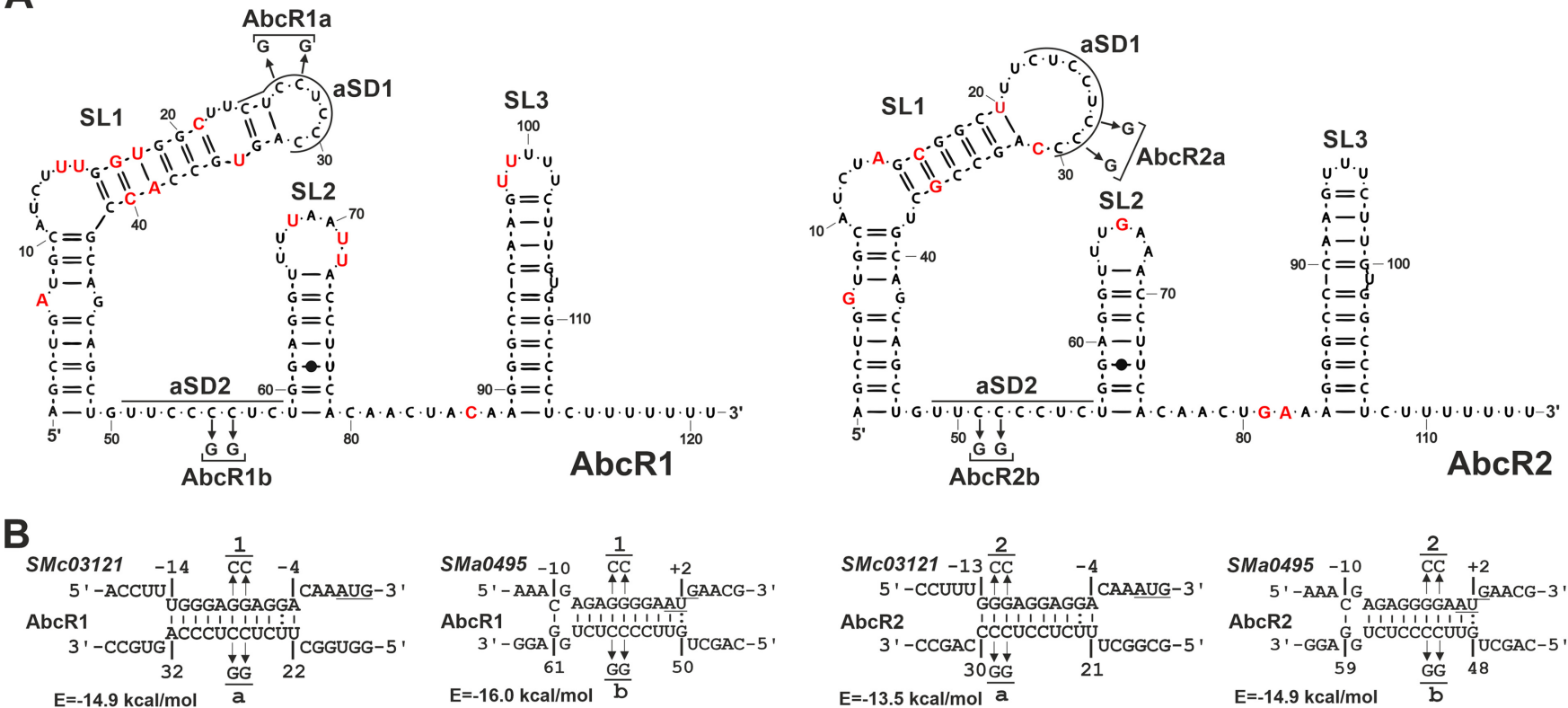

C
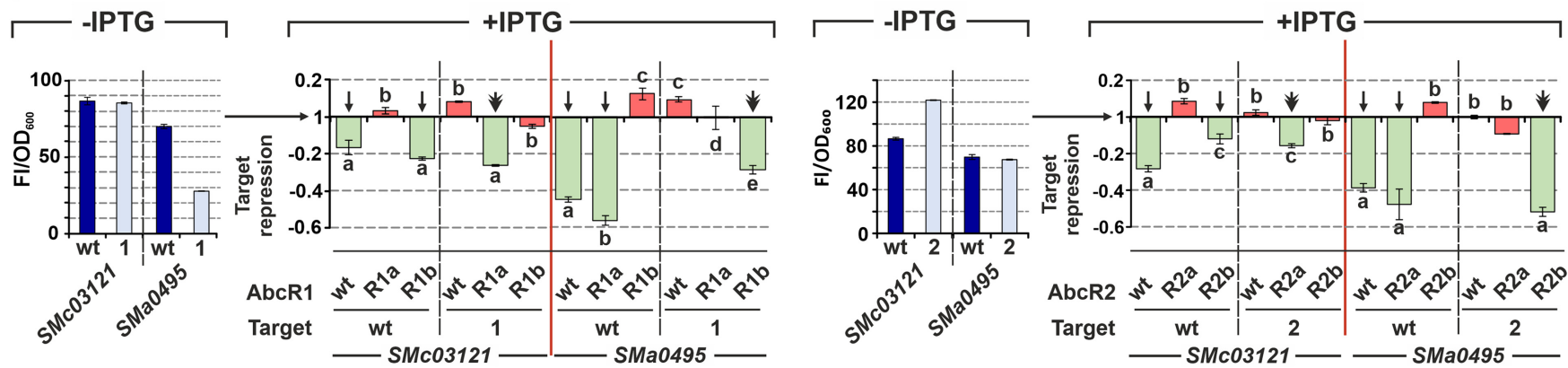

FIG 4 Genetic dissection of AbcR1/2 mRNA base-pairing interactions. (A) Predicted AbcR1 (left) and AbcR2 (right) secondary structures. Numbers indicate nucleotide positions relative to the $5^{\prime}$ end of each transcript. Stem-loops (SL) and the aSD-targeting motifs are indicated. Nucleotides differing between the sRNAs are indicated in red, while the substitutions in aSD1/2 are indicated by arrows. (B) Predicted base-pairing interactions between AbcR1/2 and the SMc03121 or SMa0495 mRNA. Numbers denote nucleotide positions relative to the AUG start codon (underlined) of the target mRNA or the $5^{\prime}$ end of the sRNA. The hybridization energy $(E)$ and nucleotide substitutions in AbcR1/2 (a/b variants) and target mRNAs (1/2 variants) are indicated. (C) Fluorescence reporter assays. Fluorescence of each reporter (wild-type and variant 1 or 2 ) in noninduced bacteria (without IPTG [-IPTG]) normalized by the OD ${ }_{600}$ of the cultures (FI/OD ${ }_{600}$ ) is presented in the blue bar graphs. The red/green bars to the right report the rates at which this basal fluorescence increased or decreased (target repression) upon IPTG induction of sRNA expression ( $24 \mathrm{~h}$ ) in strains coexpressing the target reporters with wild-type (wt) AbcR1/2 or their mutant variants, as indicated at the bottom. Plotted values correspond to means and SD of 18 fluorescence measurements, i.e., from three replicates of six double transconjugants for each reporter strain. Letters above/below bars indicate statistical groups among values from assays with each target mRNA (groups of compared values are demarcated by the red lines; analysis of variance [ANOVA] test, $P<0.05$ ). Arrows and the double arrowhead over the bars indicate the wild-type and restored non-wild-type regulation, respectively. Red bars represent no regulation.

Nucleotide changes in SMc03121 and SMa0495 leaders compensating for mutations in $\mathrm{AbcR} 1 / 2$ that abrogated target regulation did not inhibit the translation of the eGFP reporter (Fig. 4C, blue bars). However, SMa0495-1 (complementary to AbcR1b) decreased the basal fluorescence of the fusion to $\sim 40 \%$ of the wild type, most likely by interfering with the SD sequence. Remarkably, all these nucleotide substitutions fully abrogated the activities of wild-type $A b c R 1 / 2$, while restoring regulation by the complementary variants (Fig. 4C). A similar genetic dissection revealed SMa0392, SMc02417, and prbA regulation via aSD2 (Fig. S3B). SMa0392 leaders with nucleotide changes that restore pairing with $\mathrm{AbcR} 1 \mathrm{~b} / 2 \mathrm{~b}$ reduced basal-level expression of the reporters by 75 to $50 \%$ and were not regulated by the wild-type sRNAs. These mutations supported the regulation by AbcR1b/ $2 \mathrm{~b}$, as expected. Consistently with disruption of the RBS, point mutations at the predicted AbcR1/2 binding sites in SMc02417 inhibited translation (i.e., the basal activities of the reporters were scarcely $4 \%$ of the wild-type activity), thus precluding further unambiguous confirmation of aSD2 interaction at these sites. Finally, fluorescence patterns of 
A Transport $\mid$\begin{tabular}{|l} 
Sugars \\
Amino acids
\end{tabular} PHB metabolism Branched chain amino acid metabolism Vitamin biosynthesis

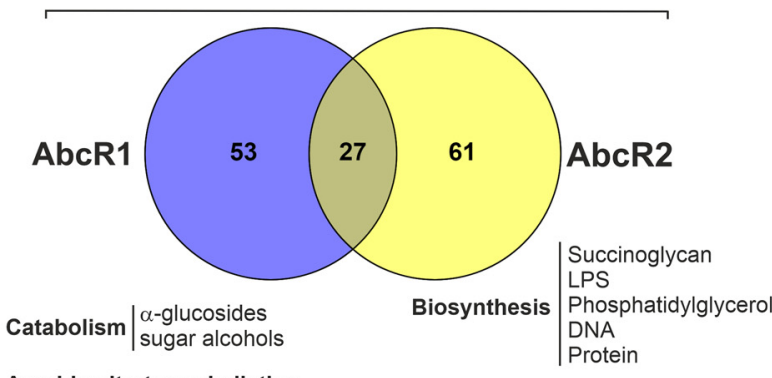

Aerobic nitrate assimilation

One carbon metabolism Microaerobic denitrification

\section{B}

\begin{tabular}{lcc|cc|} 
& \multicolumn{2}{c|}{ AbcR1 } & \multicolumn{2}{c}{ AbcR2 } \\
\cline { 2 - 6 } Carbon source & Growth rate Total flux & Growth rate & Total flux \\
\hline D-Mannitol & 0.00 & 0.00 & 0.00 & 0.00 \\
Sucrose & 0.00 & 0.00 & 0.00 & 0.00 \\
* Glycerol & 1.00 & 1.04 & 0.89 & 1.13 \\
* L-rhamnose & 0.00 & 0.00 & 0.98 & 0.94 \\
* L-Glutamate & 0.00 & 0.00 & 0.00 & 0.00 \\
\hline
\end{tabular}

C

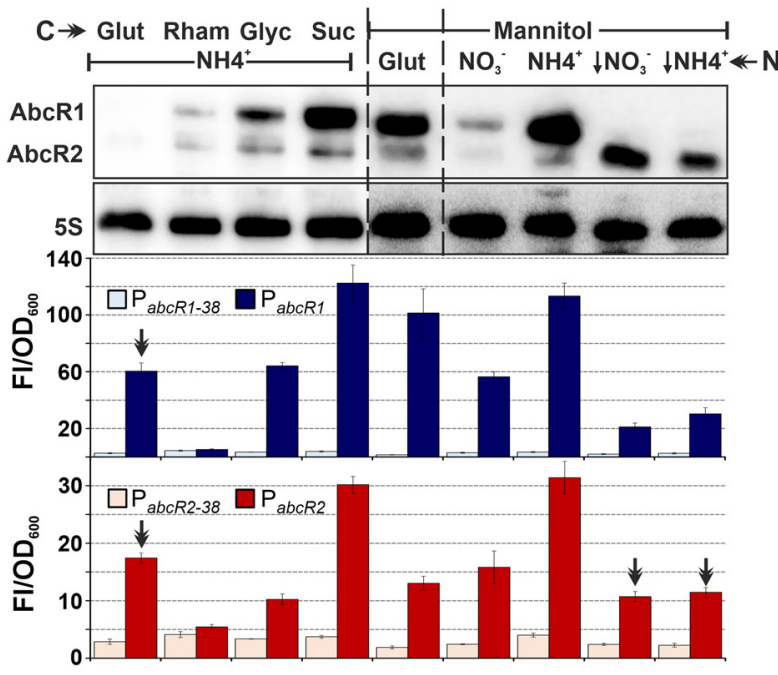

FIG 5 Metabolic model-assisted analysis of the AbcR1/2 targetomes. (A) Major transport/metabolic reactions likely regulated by AbcR1/2. The Venn diagram compares the numbers of AbcR1/2 target mRNAs represented in the model. (B) Predicted impact of target gene deletions in growth/metabolic flux in simulated defined media. Carbon sources in the media are indicated. Cells shaded red stand for the maximum impact, and those in green indicate little to no impact. Asterisks indicate matches between predictions and experimental results. (C) Changes in AbcR1/2 expression driven by shifts in carbon and nitrogen sources. Northern blot probing of total RNA from the S. meliloti Sm2B3001 strain upon growth to the onset of stationary phase in defined media with the carbon and nitrogen substrates indicated along the top. AbcR1/2 levels in mannitol-glutamate $\mathrm{MM}$ are considered the reference. Arrows indicate nitrogen stress imposed with a 0.5 mM concentration of either nitrate or ammonia. The $5 \mathrm{~S}$ rRNA was probed as an RNA loading control. Shown is the hybridization corresponding to one of two biological replicates with identical results. Bar graphs below represent fluorescence values from promoter-eGFP fusions under each growth condition determined as described in Fig. 1A. Double arrowheads indicate conditions that presumably promote AbcR1/2 posttranscriptional regulation.

wild-type and mutant prbA reporters suggest regulation by AbcR1/2 via aSD2 pairing at two contiguous sites in the mRNA leader. Wild-type sRNAs were fully active on prbA mutants, disrupting pairing with aSD2 in only one of these sites. However, these mutations were sufficient to restore regulation by $A b c R 1 b / 2 b$. This redundant targeting is reminiscent of Irp (leucine-responsive regulatory protein) regulation by GcvB in Escherichia coli (38). Collectively, these findings indicate that aSD1 and aSD2 are independent base pairing-targeting motifs that may be designed to regulate noncognate AbcR1/2 target mRNAs.

Metabolic-model-aided analysis of the AbcR1/2 targetomes. To further delineate AbcR1/2 function, we linked their regulons to the $S$. meliloti genome-scale metabolic model iGD1348, which combines core and accessory transport/metabolic reactions specified by 1,348 protein coding genes (39). Eighty (35\%) and 88 (25\%) AbcR1 and AbcR2 targets, respectively, are represented in this model, with 27 belonging to both targetomes (Fig. 5A; Data Set S1). Traits likely regulated by both sRNAs are the uptake of diverse sugars and amino acids, polyhydroxybutyrate (PHB) and branched-chain amino acids (BCAAs) metabolism, and vitamin biosynthesis. AbcR1 seems to specifically regulate the catabolism of $\alpha$-glucosides and sugar alcohols and the aerobic assimilation of nitrate in rich media. One-carbon metabolism, microaerobic denitrification, and the biosynthesis of succinoglycan (exopolysaccharide [EPS]), lipopolysaccharide (LPS), or phosphatidylglycerol are major pathways influenced by AbcR2 under abiotic stress.

We next used flux balance analysis (FBA) to predict the impact of AbcR1/2 target deletion on bacterial growth and parsimonious FBA (pFBA) to predict the requirement of a particular gene for optimal flux patterns (i.e., the total metabolic flux rate). The 
consequences of gene deletion were examined in simulated defined media differing in carbon substrates while keeping ammonia as the nitrogen source. A change of at least $10 \%$ in growth rate or total flux was considered significant (Data Set S1). Overall, these simulations predict that the combined $A b c R 1 / 2$ regulon influences $S$. meliloti transport/metabolism during growth with 64 of the $83(77 \%)$ tested carbon substrates.

Since AbcR1/2 promote posttranscriptional silencing, we expected downregulation of the sRNAs if one or more of their target mRNAs were predicted to be essential for optimal growth with a defined carbon substrate, e.g., mannitol, sucrose, glycerol, rhamnose, or glutamate (Fig. 5B). Probing of RNA from bacteria cultured in these media confirmed the predicted downregulation of $A b c R 1 / 2$ with rhamnose and glutamate, but not with mannitol or sucrose; predictions support the observed AbcR1/2 expression with glycerol (Fig. 5C). The apparent discrepancies between in silico and experimental data are likely due to the model assuming a complete loss rather than a fine-tuning of target gene expression and/or model incompleteness (i.e., transcription factors are not included, and genes specifying putative redundant transport/metabolic reactions might be missing). Although the model was not used to interrogate nitrogen metabolism, we found downregulation of both sRNAs under nitrate surplus. Nitrogen stress imposed with either ammonia or nitrate prevented AbcR1 expression while promoting AbcR2 accumulation (Fig. 5C). Fluorescence of promoter-reporter fusions revealed an overall correlation between the strength of transcription and AbcR1/2 steady-state levels under each growth condition (Fig. 5C). Exceptions were growth in glutamate/ammonia and nitrogen stress, thus hinting at posttranscriptional $\mathrm{AbcR} 1 / 2$ regulation under these conditions.

Modeling analysis predicts that AbcR1/2 expression may limit S. meliloti growth in glutamate/ammonia medium by silencing the L-amino acid ABC transporter AapJQMP. Growth kinetics in this medium confirmed that the growth rate of strain Sm2020 was reduced upon IPTG-induced expression of AbcR1/2 or their AbcR1/2a variants, but not with AbcR1/2b (Fig. 6A). Scanning of aapJQMP with IntaRNA for base pairing to AbcR1/ 2 unveiled a thermodynamically favored interaction (hybridization energy $[E]<8 \mathrm{kcal} /$ mol) with the aSD2 seed $12 \mathrm{nt}$ upstream of the start codon of aapQ, which encodes the permease of the system (Fig. 6B). This was consistent with the MAPS profiles, which suggested that this interaction might promote aapQ decay. Reverse transcriptase quantitative PCR (RT-qPCR) of RNA extracts from a similar growth experiment confirmed AbcR1/2-dependent aapQ depletion through aSD2 (Fig. 6B). All together, these data support the finding that AbcR1/2 selectively silences $S$. meliloti transport/metabolic mRNAs in response to shifts in both carbon and nitrogen substrates.

AbcR1 is required for wild-type colonization of alfalfa roots. Expression profiles suggest a prevalent AbcR1/2 activity in free-living rhizobia colonizing bulk soil or the legume rhizosphere but not in endosymbiotic bacteroids (15). Database searches identified several clusters of AbcR1/2-interacting mRNAs that are differentially expressed under rhizosphere-related conditions (i.e., exposure to alfalfa root exudates or to the nodulation gene inducer luteolin) (Fig. S4; Data Set S1). These mRNAs specify well-recognized S. meliloti metabolic traits for efficient colonization of the alfalfa rhizosphere, for example, transport/metabolism of diverse amino acids and other complex nitrogen sources and biosynthesis of the quorum-sensing autoinducers $\mathrm{N}$-acyl-homoserine lactones (AHLs) (Fig. 7A). A CopraRNA-based survey of a set of phylogenetically related genomes predicts that the regulation of orthologs of the AbcR1 target mRNAs belonging to the $S$. meliloti core genome is conserved across alphaproteobacteria interacting with eukaryotic hosts (Fig. S5). However, the occurrence of rhizosphere-related mRNA orthologs and their regulation by AbcR1 is limited to legume symbionts and even more constrained to close $S$. meliloti relatives in the case of target genes belonging to the S. meliloti accessory genome. We obtained a similar picture when AbcR2 sequences were used as queries (not shown). This conservation pattern suggests that the AbcR1/2 regulon has evolved to help alphaproteobacteria colonize the host-specific environment. These findings prompted us to investigate the impact of AbcR1/2 on the ability 
A

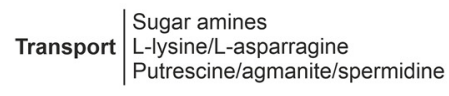

Branched chain amino acid metabolism

AHLs biosynthesis (sinR-sinl)

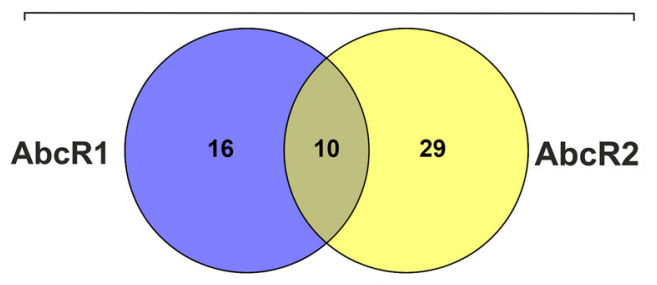

Proline catabolism Nitrogen fixation (nifA/fixK)

Purine transport and catabolism

-arginine catabolism

Microaerobic denitrification

Protein translation
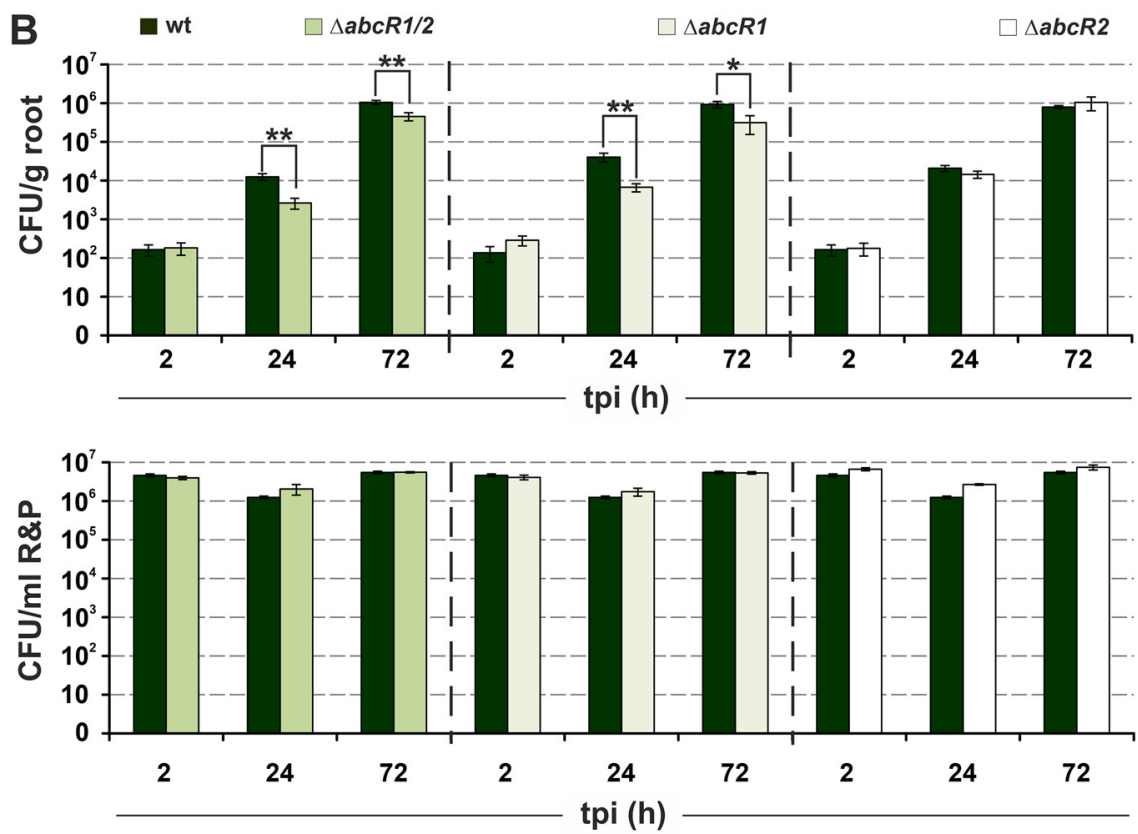

FIG 7 AbcR1 contributes to alfalfa root colonization. (A) Overlap between the AbcR1/2 targetomes and S. meliloti transcriptomic signatures under rhizosphere-like conditions. Major transport/metabolic reactions likely regulated by AbcR1/2 and relevant to rhizosphere colonization are indicated. (B) Root colonization assay. Alfalfa plants grown hydroponically were inoculated with either the wild-type Sm2B3001 strain or its single or double $a b c R 1 / 2$ deletion mutant as indicated along the top. Bar graphs represent the number of bacteria released from roots (CFU per gram of root [upper panel]) or remaining in the R\&P rooting solution (CFU per milliliter [lower panel]) at different times postinoculation (tpi). Values are means and SD of counts for a total of 45 roots from plants inoculated with each strain (three sets of 15 plants per strain treated independently). ${ }^{*}$ and ${ }^{* *}$ above the bars indicate statistically significant differences at $P$ values of $<0.05$ and $<0.005$, respectively.

(Sm2011 in which the expR gene was restored) or single or double AbcR1/2 deletion mutants. Bacterial populations either attaching to roots (cells/gram of root) or remaining in the rooting solution were then monitored by plate counting at 2,24 , and $72 \mathrm{~h}$ after plant inoculation (Fig. 7B). Counts remained invariable and equivalent among strains in the rooting solution throughout the experiment, indicating that the rooting medium does not support bacterial growth. Conversely, bacterial density on the root surface increased exponentially, indicative of active rhizoplane colonization supported by root exudates. Bacterial populations released from roots 24 and $72 \mathrm{~h}$ after inoculation were significantly lower in the $A b c R 1$ and $A b c R 1 / 2$ deletion mutants than in the wild-type strain, whereas a lack of AbcR2 did not influence colonization kinetics. FBA 


\section{Nutritionally complex environments}

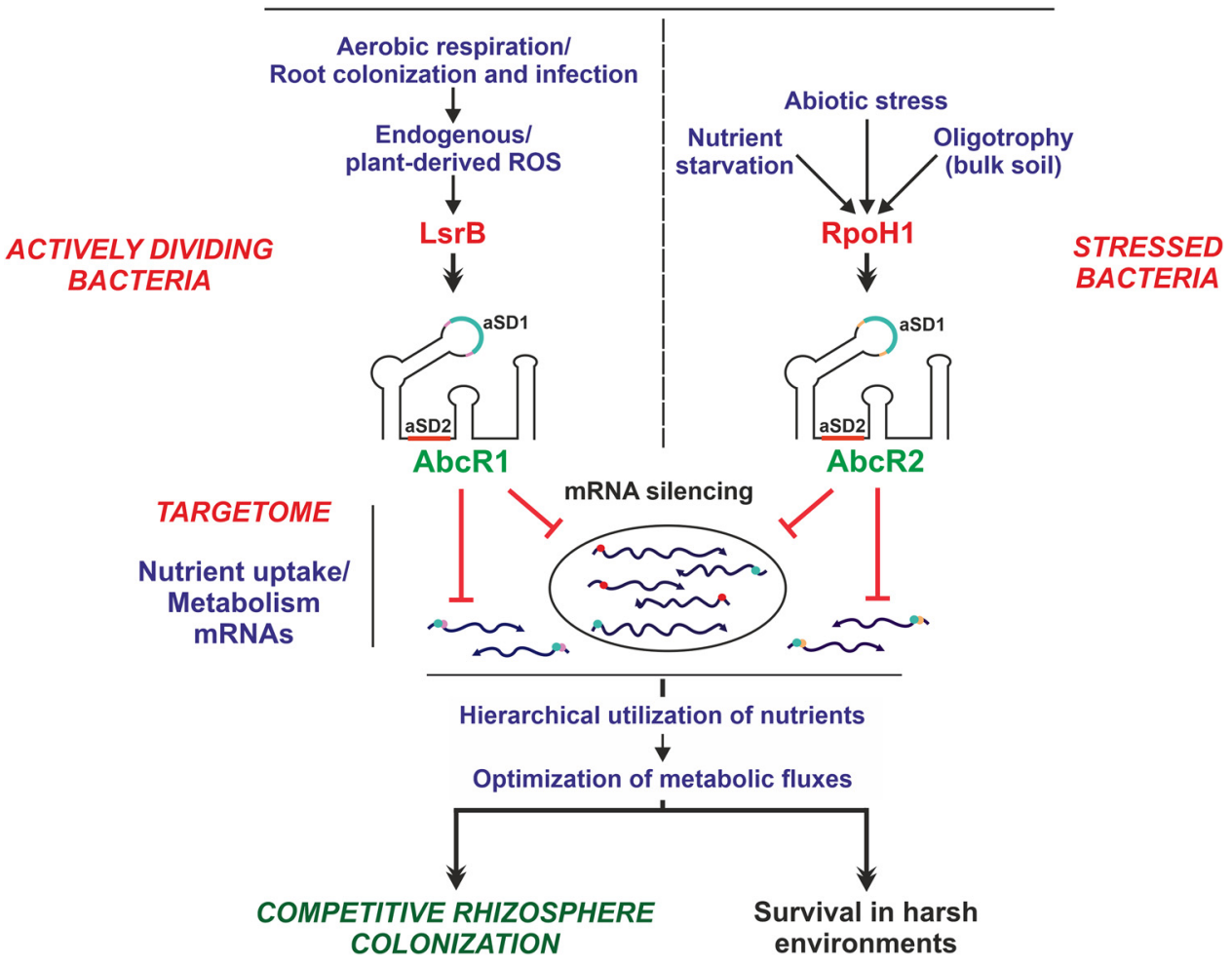

FIG 8 S. meliloti AbcR1/2 posttranscriptional regulatory network. Graphical summary of data. Details are in the text.

simulations with the metabolic S. meliloti model using a simulated rhizosphere environment similarly predicted that the AbcR1 targetome has a much greater influence on rhizosphere colonization than that of AbcR2 (Data Set S1). These data thus revealed a specific contribution of AbcR1 to alfalfa root colonization.

\section{DISCUSSION}

Base-pairing sRNAs have pivotal roles in fine-tuning the transcriptional output from regulatory networks that govern environmental adaptations in bacteria (40). However, regulation by RNA remains poorly investigated in most environmentally relevant microbes. Here, we show that the homologous S. meliloti trans-sRNAs AbcR1 and AbcR2 respond to metabolic and stress signals transduced via the LTTR LsrB and the alternative $\sigma$ factor RpoH1, respectively, to silence large and overlapping arrays of mRNAs related to nutrient uptake and metabolism (Fig. 8). Remarkably, metabolic rewiring by AbcR1 optimizes S. meliloti's ability to colonize the nutrient-rich root rhizoplane during early stages of symbiosis with its legume host. To the best of our knowledge, this is the first comprehensive genome-wide description of an RNA regulatory network controlling a major adaptive trait in nitrogen-fixing legume symbionts, a group of soil bacteria essential for planet sustainability.

LsrB and RpoH1 trace independent input modules for the AbcR1/2 network. SRNA abundance is regulated primarily at the level of transcription initiation (41). Supporting in silico predictions, genetic and biochemical approaches unequivocally identified LsrB with the $\sigma$ factor RpoH1 as the major regulators of S. meliloti AbcR1 and AbcR2 transcription, respectively. These findings confirm that the regulation of AbcR1 is conserved in alphaproteobacteria (28-30). S. meliloti LsrB senses the concentration of reactive oxygen species (ROS) that may derive from either aerobic respiration (endogenous ROS), redox-cycling compounds secreted by neighboring soil organisms, or the oxidative bursts of plant defense responses during symbiosis $(42,43)$. Redox signal transduction by LsrB boosts the transcription of genes for the biosynthesis of LPS and ROS 
scavenging systems (e.g., glutathione), thereby preventing cell damage, root infection arrest, and premature nodule senescence $(44,45)$. Thus, plant-derived ROS is likely the biotic signal that drives AbcR1 transcription in undifferentiated rhizobia at early symbiotic stages $(15,23,46)$. Our data further suggest that LsrB might also transduce shifts in carbon and nitrogen metabolism in free-living S. meliloti bacteria (Fig. 5C), possibly by sensing the differential accumulation of endogenous ROS. Unlike that of AbcR1, AbcR2 regulation had not previously been investigated in alphaproteobacteria. A microarray-based transcriptome profile of $S$. meliloti $r p o H$ mutants revealed differential expression of a few annotated trans-sRNAs but overlooked the downregulation of AbcR2 (34). RpoH1 recognizes gene promoters that respond to diverse stressors, such as heat shock, salinity, nutrient starvation, or the plant intracellular milieu (34), which is consistent with the stress-induced transcription of AbcR2 in free-living bacteria (see Fig. S1 in the supplemental material).

In S. meliloti, a lack of either $\mathrm{LsrB}$ or $\mathrm{RpoH} 1$ results in severe growth and endosymbiotic phenotypes, hinting at their seemingly constitutive activity, which only partially explains the differential AbcR1/2 expression (e.g., AbcR2 is not detected in nodules) $(34,47,48)$. Thus, posttranscriptional and/or posttranslational modifications of LsrB and RpoH1 might be further determinants of $A b c R 1 / 2$ transcription rates in free-living and symbiotic rhizobia (49). In this regard, it is known that the strength of LsrB regulation depends on the oxidation of two cysteine residues that promote protein dimerization via disulfide bonds (42). The extent to which this posttranslational modification influences LsrB affinity for promoter binding and $A b c R 1$ transcription must be investigated.

MAPS-derived insights into the AbcR1/2 network. The identification of regulatory motifs in trans-sRNAs and their mRNA targets remains challenging $(50,51)$. Computational tools typically predict large sets of target mRNA candidates for a trans-sRNA, but the limited complementarity between the partners often leads to exceedingly high false-positive prediction rates (52). This also applies to S. meliloti AbcR1/2, although previous genetic reporter assays confirmed as targets a few mRNA candidates predicted in silico $(15,22)$. Even though these assays are suitable to validate and dissect sRNA-mRNA interactions, they do not provide genome-wide insights into the regulation occurring endogenously under specific environmental conditions. Thus, we chose MAPS to tackle the comprehensive profiling of the S. meliloti AbcR1/2 mRNA interactomes under growth conditions that stimulate endogenous upregulation of each sRNA (53). Remarkably, roughly $6 \%$ of S. meliloti mRNAs were identified in the combined AbcR1/2 targetome, most of which encode nutrient uptake, catabolism, or biosynthesis functions (Data Set S1). AbcR1/2 thus resemble $E$. coli GcvB sRNA in regulating an exceptionally large number of metabolic genes $(54,55)$. AbcR1/2 are core components of the S. meliloti pangenome (24), but many of their putative mRNA targets are Hfq partners, belong to the accessory genome, and/or are encoded in the pSymB megaplasmid (Fig. 2). This suggests a major impact of these sRNAs in the effective integration of acquired adaptive metabolism into core regulatory networks $(22,56)$.

MAPS captures sRNA-mRNA base pairing, but it is not inherently designed to inform about the impact of these interactions on target mRNA stability. However, the markedly uneven distribution of sequencing reads over large sets of mRNAs copurified with tagged AbcR1/2 suggests accelerated decay of the targets upon base pairing, thereby providing interaction signatures for regulation (Fig. 3). In S. meliloti, the set of ribonucleases and degradosome-like assemblies containing $\mathrm{Hfq}$ are poorly characterized (13, $35,57)$. Our MAPS setup might report on the turnover dynamics of the AbcR1/2 mRNA interactomes if sRNA baits are expressed in the relevant RNase knockout mutants.

Interaction signatures inferred from the recovery profiles of the AbcR1/2 target mRNAs and further genetic approaches suggest that AbcR1/2 act predominantly by a canonical Hfq-dependent mechanism relying on base pairing at the RBS leading to translation inhibition (21) (Fig. 3 and 4 and Fig. S3). Nonetheless, our data also envisage minor but plausible alternative modes of action independent of $\mathrm{Hfq}$ or involving 
interactions in the coding sequence of the target mRNAs (Fig. 3). The latter has already been shown for the $A$. tumefaciens AbcR1/2 homologs (31). Reporter assays confirmed AbcR1/2 regulation of a set of three newly identified targets (SMc02417, SMc03121, SMa0392) that all code for ABC transport proteins (Fig. S3). These experiments provided further evidence that the aSD seeds (aSD1/2) are major motifs involved in mRNA targeting. aSD1 and aSD2 differ slightly in their nucleotide sequences, and they were genetically shown to fulfil independent targeting roles (Fig. 4). A few of the nucleotides that flank aSD1 differ between AbcR1 and AbcR2, which might provide specificity for targeting. Indeed, we previously showed specific AbcR1-mediated silencing of livK most likely through aSD1 (15). Conversely, aSD2 is embedded within an ultraconserved nucleotide stretch and presumably supports the regulation of common target sets. Therefore, it seems likely that the functional specificity of AbcR1 and AbcR2 is conferred largely by their differential expression rather than by their targeting potential.

A metabolic model delineated the adaptive functions of AbcR1/2. In S. meliloti, many predicted transport or metabolic reactions have scarce experimental support. A metabolic-model-assisted analysis of the targetomes charted by MAPS suggested that AbcR1/2's impact extends beyond primary carbon/nitrogen energy pathways to the regulation of biosynthesis of the major carbon storage polymer PHB or cell envelope components (e.g., EPS, LPS, phosphatidylglycerol) (Fig. 5). In S. meliloti, PHB biosynthesis is negatively regulated by the MmgR sRNA under carbon surplus conditions (19). MmgR is repressed by the global carbon flow regulator AniA $(58,59)$, which is a putative target of $A b c R 1 / 2$ regulation. Thus, AniA may serve as a connection node of the MmgR and AbcR1/2 regulatory networks for the robust control of carbon homeostasis. mRNAs specifying cell wall synthesis were identified as AbcR2-specific targets. Therefore, AbcR2 might play a role in the regulation of cell envelope remodeling in response to different stresses (60-62). The model also linked AbcR1/2 targets to nitrate assimilation and denitrification pathways, which was further supported by profound changes in AbcR1/2 expression upon shifts in the quality and quantity of the nitrogen source (Fig. 5C). RNA regulation of nitrogen metabolism has been reported in free-living nitrogen fixers but not in S. meliloti (63-65).

This approach was useful in predicting AbcR1/2 targets required for growth in defined media formulated with specific carbon substrates. As a proof of principle, we demonstrated AbcR1/2-mediated silencing of the permease component of the L-amino acid transporter AapJQMP (Fig. 6). Together with the BraDEFGC transport system, AapJQMP rescues the symbiotic autotrophy for BCAAs of Rhizobium leguminosarum bv. viciae bacteroids within indeterminate pea nodules, which is not a feature of alfalfa nodules $(66,67)$. Regulation of $a a p Q$ by $A b c R 1 / 2$ is predicted to be conserved in $R$. leguminosarum and may have a specific impact on pea nodule metabolism that merits further investigation.

A target-centric perspective of AbcR1 contribution to root colonization. Root exudates make the rhizosphere and rhizoplane nutrient-rich but strongly selective environments for the root microbiome $(3,68)$. The AbcR1 knockout phenotype thus suggests that RNA regulation of metabolism provides $S$. meliloti with a competitive advantage for host root colonization and saprophytic long-term survival in the rhizosphere. Both the reported specific contribution of AbcR1 to S. meliloti growth in complete media and this novel phenotype are consistent with AbcR1 levels far exceeding AbcR2 levels when both sRNAs are cotranscribed in rhizobia actively dividing under nutrient surplus or at preinfection symbiotic stages (15). Expression and targetome profiles of AbcR2 predict similar impacts of this SRNA in the colonization of bulk soil or the rhizosphere under harsh environmental conditions.

The AbcR1/2 interactomes are well represented in the transcriptomic signatures of rhizospheric S. meliloti bacteria (Fig. S4), which are enriched in transport/metabolic genes for the utilization of amino acids, sugar amines, and polyamines (69). Interestingly, mariner-based transposon insertion sequencing has recently uncovered that knockout of genes encoding uptake systems for quaternary amines, BCAAs, Lamino acids (e.g., aapJQMP), opines, and polyamines enhances $R$. leguminosarum bv. 
viciae fitness in pea rhizosphere (2). Similarly, downregulation of the $\alpha$-glucoside/trehalose/maltose transporter agIE, also identified as AbcR1/2's target, switches the metabolism of Ensifer spp. to the utilization of plant-derived dicarboxylic acids in a disaccharide-rich bulk soil to favor nodulation of pigeon pea (70). Competitive colonization of rhizosphere and other nutritionally complex environments by rhizobia presumably demands optimization of metabolic fluxes through the hierarchical utilization of available substrates. Massive but controlled silencing of metabolic mRNAs from the LsrB and $\mathrm{RpoH} 1$ regulons would help prevent energy-expensive uptake, catabolism, and biosynthesis of nonpriority compounds. Such a large RNA network provides additional levels of regulation relying on mRNA competition for the sRNA. First, computational predictions suggest that the binding affinity between the sRNA and the target mRNAs is a determinant of the hierarchy in the network; i.e., more extensive base pairing to the sRNA would provide priority for regulation (40). Second, competing endogenous RNAs (ceRNAs) acting as sRNA antagonists can also mediate cross-regulation of mRNAs (71). Crosstalk between ABC transporter mRNAs via a target mRNA-derived ceRNA has already been demonstrated in the GcvB regulon (72). Therefore, this is a plausible mechanism for the posttranscriptional control of AbcR1/2 levels, which likely occurs upon specific metabolic shifts or in endosymbiotic bacteria. Our MAPS data set can be further inspected to search for such AbcR1/2 sponges (73).

To conclude, our findings depict a singularly large RNA network that governs metabolic adaptations of $S$. meliloti during colonization of the selective alfalfa rhizosphere. Similar networks have likely diverged to help alphaproteobacteria adapt to their specific host-associated soil environments. Since AbcR1/2-targeting motifs are potentially modifiable to base pair and regulate noncognate mRNAs, this network might be rewired at different levels to engineer highly competitive biofertilizers.

\section{MATERIALS AND METHODS}

Bacterial strains and growth conditions. Bacterial strains and plasmids used in this work, along with their relevant characteristics, are listed in Table S1 in the supplemental material. E. coli strains were routinely grown in lysogeny broth (LB) medium at $37^{\circ} \mathrm{C}$, and rhizobia were grown in either complex tryptone-yeast (TY) medium (74) or defined mannitol-glutamate MM (75) at $30^{\circ} \mathrm{C}$. To assess the stress-dependent expression of AbcR1/2, exponentially growing bacteria in MM were cultured for a further $1 \mathrm{~h}$ upon salt $(400 \mathrm{mM} \mathrm{NaCl})$ and heat $\left(40^{\circ} \mathrm{C}\right)$ shocks. To test the effect of shifts in nitrogen metabolism on AbcR1/2 accumulation, the L-glutamate $(6.5 \mathrm{mM})$ of the standard $\mathrm{MM}$ was replaced by $\mathrm{NH}_{4} \mathrm{Cl}(10$ or $0.5 \mathrm{mM}$ ) or $\mathrm{KNO}_{3}$ (10 or $0.5 \mathrm{mM}$ ). Similarly, the impact of different carbon sources in AbcR1/2 expression was assessed in $\mathrm{MM}$ with ammonia $(10 \mathrm{mM})$ as the nitrogen source and either mannitol (54 $\mathrm{mM})$, sucrose (10 $\mathrm{mM})$, glycerol $(15 \mathrm{mM})$, glutamate $(6.5 \mathrm{mM})$, or rhamnose $(15 \mathrm{mM})$ as the carbon substrate. When required, growth media were supplemented with the appropriate antibiotic(s) (in micrograms per milliliter): streptomycin (Sm) at 480, tetracycline (Tc) at 10, erythromycin ( $\mathrm{Er}$ ) at 100, and kanamycin (Km) at 50 for E. coli and at 180 for S. meliloti.

Oligonucleotides. Sequences of the oligonucleotides used as probes for Northern hybridization or as amplification primers for cloning and RT-qPCR are listed in Table S2.

RNA isolation and Northern blot analysis. Total RNA was isolated from free-living bacteria cultured under the described conditions by acid phenol-chloroform extraction (76). For Northern analysis, RNA samples (15 to $20 \mu \mathrm{g}$ ) were subjected to electrophoresis on 6\% polyacrylamide-7 M urea gels, blotted into nylon membranes, and probed with $5^{\prime}$-end-radiolabeled PbAbcR1/2 or PbAbcR2 oligonucleotides as described previously (23).

Construction of S. meliloti mutants. Knockout mutants were generated by deletion of the wildtype loci using the suicide plasmid pK18mobsacB to induce allelic replacement by double crossover $(56$, 77). Plasmids were mobilized to the parent strains by biparental mattings (78). $\mathrm{Sm} \Delta / \mathrm{sr} B$ was generated in Sm2011 by a markerless in-frame deletion of the IsrB coding sequence using pK18 $\Delta / s r B$. To construct pK18 $\Delta / s r B, 822-b p$ and 814-bp DNA fragments flanking the /srB open reading frame (ORF) were amplified from genomic DNA with the EcoRluplsrB/BamHIATGIsrB and BamHITGAlsrB/XbaldownlsrB primer pairs. $P C R$ fragments were digested with EcoRI/BamHI and BamHI/Xbal, respectively, and ligated to the pK18mobsacB EcoRI and Xbal restriction sites, leading to insertion of the tandem fragments via their common BamHI site. Sm2020 (triple abcR1 abcR2 nfeR1 deletion mutant) was generated in Sm2019 (derived from Sm2011) (Table S1) by successive replacement of the three sRNA loci by a 135-bp erythro-

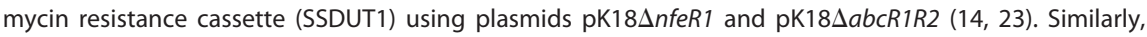
$\mathrm{Sm} \Delta a b c R 1, \mathrm{Sm} \Delta a b c R 2$, and $\mathrm{Sm} \Delta a b c R 1 R 2$ were generated with the parent strain Sm2B3001 (derived also from Sm2011) (Table S1) using plasmids pK18 $a b c R 1$, pK18 $a b c R 2$, and pK18 $a b c R 1 R 2$ (15, 79). All PCR amplifications required for cloning were performed with the proofreading Phusion high-fidelity DNA polymerase (Thermo Scientific). Plasmid inserts were always checked by sequencing to confirm the 
absence of PCR-introduced mutations. The mutants $\operatorname{Sm} \Delta / s r B$, Sm $\Delta a b c R 1, \operatorname{Sm} \Delta a b c R 2$, and $\operatorname{Sm} \Delta a b c R 1 R 2$ were further checked by whole-genome sequencing.

Construction of plasmids for induced AbcR1/2 expression and tagging. For the IPTG-induced expression of wild-type and MS2 aptamer-tagged AbcR1/2, we constructed plasmids pSKiAbcR1, pSKiAbcR2, pSKiMS2AbcR1, and pSKiMS2AbcR2, which are based on the indirect sinR-sinl system as described previously (18). The promoter region $\sin R-P_{\sin l}-T S S_{\sin l}$ was $P C R$ amplified using genomic DNA as the template with the primers sinR_NfelF/TSS3_28bp_b_sinIR, and the MS2 aptamer was amplified from pSRKMS2 using the MS2FusTSSI/Hindlllvec primer pair (35). These two fragments overlap and were jointly used as the template for amplification with sinR_NdelF/Hindlllvec, and the resulting PCR product was restricted with Ndel and Xbal and inserted into pSRKKm to yield pSKiMS2 (80). AbcR1 and AbcR2 were amplified from pSRKMS2AbcR1 or pSRKMS2AbcR2 (constitutively expressing tagged AbcR1 or AbcR2) using the PCR1/PCR2 primers (35). PCR products were digested with Xbal and Xhol and inserted into pSKMS2 to generate pSKiMS2AbcR1 and pSKiMS2AbcR2. Alternatively, AbcR1 and AbcR2 were amplified from pSRK-R1 or pSRK-R2 (constitutively expressing the wild-type transcripts) using the AbcR1OexfusTSSI/secSRK or AbcR2OexfusTSSI/secSRK primer pairs, respectively (15). Both forward primers contain a sequence complementary to TSS3_28bp_b_sinIR. The first PCR products were used as the template for a second PCR using the primer pair sinR_NdelF/Hindlllvec or sinR_NdelF/secSRK. The resulting fragments were restricted with $\mathrm{Ndel}$ and Xbal and inserted into pSRKKm to generate pSKiAbcR1 and pSKiAbcR2.

Replacements of specific nucleotides within aSD1/2 were performed using a two-step PCR strategy based on overlapping fragments using pSKiAbcR1 or pSKiAbcR2 as the template, as described previously (14). The first round of PCR amplifications was performed with sinR_NdelF or secSRK (both hybridizing to all plasmid templates) and their respective primer pair carrying the desired mutations (Table S2). Each pair of complementary PCR products was used as the template in the second PCR with sinR_NdelF/ secSRK. The resulting products were digested with Ndel/Xbal and ligated to pSRKKm to yield plasmids pSKiAbcR1a, pSKAbcR1b, pSKAbcR2a, and pSKAbcR2b, which were mobilized to $S$. meliloti strain Sm2020 by biparental mattings.

MS2 affinity purification coupled with RNA sequencing (MAPS). The affinity purification assays were performed by following a previously described protocol adapted to S. meliloti $(32,33,35,36)$. Sm2020 cells carrying pSKiMS2AbcR1 or pSKiAbcR1 (control of column-binding specificity) were grown in TY and MM to exponential phase. Bacteria carrying pSKiMS2AbcR2 or the control pSKiAbcR2 were cultured in TY and MM to stationary phase or subjected to temperature and salt upshifts upon growth in TY to exponential phase. Aliquots of wild-type- and tagged-AbcR1/2-derived cultures were independently pooled, and cells equivalent to an optical density at $600 \mathrm{~nm}\left(\mathrm{OD}_{600}\right)$ of 200 were harvested by centrifugation $\left(4^{\circ} \mathrm{C}\right)$ at $3,500 \times \mathrm{g}$ for $15 \mathrm{~min}$ after the addition of IPTG $(1 \mathrm{mM})$ to induce sRNA transcription. Cells were washed with $20 \mathrm{~mL}$ TE buffer $(\mathrm{pH}$ 8), centrifuged again, resuspended in $4 \mathrm{~mL}$ lysis buffer (20 mM Tris- $\mathrm{HCl}, \mathrm{pH} 8.0,150 \mathrm{mM} \mathrm{KCl}, 1 \mathrm{mM} \mathrm{MgCl}, 1 \mathrm{mM}$ dithiothreitol [DTT]), and broken using a French press. Soluble cell fractions were subjected to affinity chromatography on MS2-MBP-conjugated amylose resin as described previously $(35,36)$. Eluted RNA was isolated by phenol-chloroform extraction followed by precipitation of the aqueous phase with $4 \mathrm{vol}$ ethanol (EtOH) in the presence of $20 \mu \mathrm{g}$ of glycogen. To monitor the procedure, RNA was obtained from $50 \mu$ l of cleared lysate, flow through, and wash fractions by acid phenol/chloroform extraction (76). These RNA preparations were probed with $\mathrm{PbAbcR} 1 / 2$ upon Northern blotting.

Strand-specific cDNA libraries from RNA fractions eluted from columns were generated and sequenced in the Illumina NextSeq Mid 150 platform. Demultiplexed sequencing reads were mapped with Bowtie2 v2.2.3 using parameters standard to the S. meliloti Sm1021 reference sequence downloaded from the RhizoGATE portal $(37,81)$. Uniquely mapped reads were assigned to protein-coding genes or noncoding RNAs with Rsubread 3.12 (82). Read counts for each genomic feature were normalized by coverage, and the resulting numbers of reads per kilobase per million (RPKM) were the basis for fold change calculations (83). The Integrative Genomics Viewer (IGV) software was used for data visualization (84).

Fluorescence reporter assays. The transcriptional fusions reporting promoter activity were generated in the promoterless vector pBBeGFP (14). AbcR1 (334 bp) and AbcR2 (206 bp) promoters were amplified with the primer pairs XbalAbcR1/PC15Rv and EcoRIPC16/PC16Rv, respectively. The PCR products were digested with Xbal $\left(\mathrm{P}_{a b c R 1}\right)$ or HindllI/Xbal $\left(\mathrm{P}_{a b c R 2}\right)$ and cloned into pBBeGFP to generate pBBAbcR1::eGFP and pBBAbcR2::eGFP. Trimmed versions of both promoters (38 bp) were generated by annealing the oligonucleotides PR1_50i/PR1_50 $\left(\mathrm{P}_{a b c R 1-38}\right)$ and PR2_58i/PR2_58 $\left(\mathrm{P}_{a b c R 2-38}\right)$ and cloning the products into pGEM-T. $\mathrm{P}_{A b C R 1-38}$ and $\mathrm{P}_{A b C R 2-38}$ were retrieved from $\mathrm{pGEM}-\mathrm{T}$ by Spel-Xbal restriction and finally inserted in pBBeGFP to yield pBBAbcR1-38::eGFP and pBBAbcR2-38::eGFP.

Reporter fusions of SMC02417 and SMa0392 to eGFP were generated in plasmid pR-eGFP (15). For this, genomic regions of SMC02417 and SMa0392 from their respective transcription start sites to the 12 th or 77 th codons were amplified with the a0392F/a0392R and c02417F/c02417R primer pairs, respectively. The resulting PCR products were digested with BamHI/Nhel and cloned into pR-eGFP to yield pRSMc02417::eGFP and pRSMa0392::eGFP. Compensatory nucleotide substitutions in all tested target mRNAs (i.e., SMc03121, SMa0495, prbA, SMc02417, and SMa0392) for regulation by the corresponding $A b c R 1 / 2$ variants were introduced by a two-step PCR using the respective wild-type reporter fusion as the template. The first round of PCR amplifications was performed with PCR2 or Egfp-139_rev (both hybridizing to the plasmid templates) and their respective primer pair carrying the specific mutations (Table S2). Each pair of complementary PCR products was used as the template in the second PCR with PCR2/Egfp-139. The resulting products were digested with BamHI/NdeHI and ligated to pR-eGFP to 
generate the new set of reporters ( 1 and 2 variants of each wild-type reporter). All reporter plasmids were transferred by biparental conjugation to Sm2020 harboring plasmids expressing either wild-type AbcR1/2 or their a/b variants. Transconjugants for each RNA-target fusion combination were grown to exponential phase $\left(\mathrm{OD}_{600}\right.$ of 0.2 to 0.3$)$, divided into untreated and $0.5 \mathrm{mM}$ IPTG-treated cultures, and incubated for $24 \mathrm{~h} . \mathrm{OD}_{600}$ and fluorescence (excitation, $485 \mathrm{~nm}$; emission, $520 \mathrm{~nm}$ ) were measured in a Thermo Scientific Varioskan LUX multimode microplate reader. Fluorescence values were normalized to the culture $\mathrm{OD}_{600}$.

Electrophoretic mobility shift assays (EMSA) with LsrB. The LsrB coding sequence was PCR amplified from genomic DNA using the primers LsrB_Fw_ndel/LsrB_Rv_BamHI and cloned into the vector pET-16b (Novagen) between the Ndel/BamHI restriction enzymes sites, yielding p16LsrB encoding a Histagged LsrB. Recombinant LsrB was produced and purified as described previously (35). The EcoRIPC16/ PC16Rv and XbalAbcR1/PC15Rv primer pairs were used to amplify $P_{a b c R 1}(334 \mathrm{bp})$ and $P_{a b c R 2}(206 \mathrm{bp})$, respectively, which were further purified from agarose gels with the GFX PCR DNA and Gel Band purification kits (GE Healthcare). Binding reactions were performed with $100 \mathrm{nM}$ radiolabeled probes in the absence or presence $(1 \mu \mathrm{M})$ of purified LsrB and then subjected to electrophoresis and analyzed with the Personal FX equipment and Quantity One software (Bio-Rad) as described previously (85).

RT-qPCR. RNA samples obtained as described previously were further cleaned up with the RNeasy minikit (Qiagen) following DNase digestion. CDNA was synthesized with the qScript cDNA synthesis kit (Quantabio). RT-qPCR was carried out in a QuantStudio 3 system (Thermo Fisher Scientific) using the Takyon low Rox SYBR $2 \times$ master mix blue dTTP (Eurogentec). The ratios of transcript abundance were calculated as the $\Delta \Delta C T$ (where $C T$ is threshold cycle) mean average of results from three replicates using three independent RNA extracts, where the $\Delta \Delta C T$ represents the level of gene expression in the IPTGinduced strain relative to that in the untreated control strain. The seemingly constitutive gene SMc01852, encoding a phosphofructokinase, was used to normalize gene expression (86). Control reactions without reverse transcriptase (-RT) in the RNA samples were simultaneously performed to confirm an absence of DNA contamination.

Root colonization assays. Alfalfa (Medicago sativa L. "Aragón") plants were sterilized, germinated, and grown in hydroponic cultures under axenic conditions as described previously (87). Root colonization by bacteria was assessed by counting CFU. Rhizobial strains were grown on TY plates at $30^{\circ} \mathrm{C}$ for 2 days, and the cell mass was resuspended in TY broth to an $\mathrm{OD}_{600}$ of 0.5 and then diluted 100 -fold in sterile water to prepare an inoculum of approximately $10^{6}$ bacterial cells/plant. At defined times, 15 roots inoculated with each rhizobial strain were washed 3 times with $20 \mathrm{~mL}$ sterile water to remove the loosely attached bacteria, and the roots were weighed in groups of five placed into 2-mL Eppendorf tubes. Then, $1 \mathrm{~mL}$ of sterile TE buffer was added to each tube, and the attached cells were released by two sonication pulses of $1 \mathrm{~min}$ each in an Ultrasons sonicator bath with a pause time of 1 min between the pulses. Cells were subsequently quantified by counting CFU (normalized to grams of root). Experiments were conducted in triplicate for each tested strain.

Computational methods. Promoter sequence alignments were generated with ClustalW implemented in BioEdit (88), and searches for conserved motifs were done with the MEME algorithm (89; http://meme-suite.org/index.html). The logo of the motif consensus sequence was generated at http:// weblogo.berkeley.edu/logo.cgi. Venn diagrams were generated with the Venny 2.0 tool (https:// bioinfogp.cnb.csic.es/tools/venny/index2.0.2.html). CopraRNA (v 2.1.2) and IntaRNA (v 3.2.0; http://rna .informatik.uni-freiburg.de/) were used to predict sRNA-mRNA base-pairing interactions $(52,90)$.

FBA simulations were performed in MATLAB R2019a (MathWorks) using SBMLToolbox version 4.1.0 (91), libSBML version 5.17 (92), scripts from the COBRA Toolbox commit 6a99a1e (93), and the iLOG CPLEX Studio 12.9 .0 solver (ibm.com). All analyses were performed on the S. meliloti metabolic model iGD1348 (39). For each nutrient condition that was tested, the maximal growth rate and the overall metabolic flux rates of iGD1348 were determined using the "optimizeCbModel" and "pFBA" functions of the COBRA Toolbox. Then, each gene belonging to the AbcR1/2 targetome was either individually or simultaneously deleted, and the maximal growth rate and the overall metabolic flux rates were determined. $\mathrm{AbcR} 1 / 2$ were predicted to regulate transport/metabolism during growth with a carbon source when deletion of any individual gene or the entire targetome resulted in a growth rate of $<90 \%$ of that of the wild-type model or an overall flux rate of $>110 \%$ that of the wild-type model. Analyses in simulated bulk soil and rhizosphere conditions were performed using previously defined nutritional conditions (94). Code to repeat the analyses is provided as Text $\mathrm{S} 1$.

The 23 complete S. meliloti genomes present in the ftp NCBI folder on 17 February 2021 were downloaded, and the pangenome was computed with the Pan/Core-Genome and the Gene Phyloprofile tools of the MicroScope platform (95), with thresholds of $80 \%$ amino acid identity and $80 \%$ alignment coverage, as previously reported (96). The list of AbcR1/2 target genes was used as a query to investigate the pattern of expression in S. meliloti transcriptomic data from cultures treated with alfalfa root exudates and luteolin (69) as a proxy of rhizospheric conditions.

Data availability. Raw RNA-seq data can be accessed at the SRA database (BioProject ID PRJNA735891).

\section{SUPPLEMENTAL MATERIAL}

Supplemental material is available online only.

TEXT S1, TXT file, $0.03 \mathrm{MB}$.

FIG S1, TIF file, 1.1 MB. 
FIG S2, TIF file, $2 \mathrm{MB}$.

FIG S3, TIF file, 2.3 MB.

FIG S4, TIF file, $1 \mathrm{MB}$.

FIG S5, TIF file, 0.8 MB.

TABLE S1, DOCX file, $0.02 \mathrm{MB}$.

TABLE S2, DOCX file, $0.02 \mathrm{MB}$.

DATA SET S1, XLSX file, 2.7 MB.

\section{ACKNOWLEDGMENTS}

This work was supported by grants BFU2017-82645-P and PID2020-114782GB-I00, funded by MCIN/AEI/10.13039/501100011033 and by "ERDF A way of making Europe" (BFU2017-82645-P), and grant P20_00185, funded by the Junta de Andalucía PAIDI/ FEDER/EU, all awarded to J.I.J.-Z. This work was also supported by grant FPDI-201316255, funded by MCIN/AEI/10.13039/501100011033 ("Formación Post-doctoral" Juan de la Cierva program) to M.R., and an FPU fellowship (FPU16/01275) from the Ministerio de Universidades to N.I.G.-T. A.P. was a recipient of a CSIC Fellowship in the frame of the JAEPre Program (JAEPre_09_00145). Research in the G.C.D. laboratory is supported by a Natural Sciences and Engineering Research Council of Canada (NSERC) Discovery Grant. A.M. is supported by the "MICRO4Legumes" grant from the Italian Ministry of Agriculture. The funders had no role in study design, data collection and interpretation, or the decision to submit the work for publication.

Alicia Barroso (Genomics Unit of Instituto de Parasitología y Biomedicina LópezNeyra, CSIC, Granada) is acknowledged for RNA-seq. We thank Eduardo Andrés-León and Laura C. Terrón from the same institute for assistance in the bioinformatics analysis, Fernando M. García-Rodríguez for help in LsrB purification, María D. Molina-Sánchez for advice on the RT-qPCR experiments, and Sharon R. Long and Melanie Barnett (Stanford University) for providing us with the S. meliloti rpoH strains.

N.I.G.-T. performed most of the experiments, analyzed data, and wrote the manuscript. M.R. planned and initiated experiments, discussed and interpreted results, and critically read the manuscript. G.C.D. performed metabolic model analysis, aided in the interpretation of results, and critically read the manuscript. A.M. conducted database searches, aided in the interpretation of results, and critically read the manuscript. V.M. constructed some S. meliloti mutants and aided in Northern blot experiments. A.P. performed some initial experiments. A.U. performed part of the fluorescence reporter assays with AbcR2. J.I.J.-Z. conceived and designed the study, aided in experiments, analyzed data, and wrote the manuscript.

\section{REFERENCES}

1. Fowler D, Coyle M, Skiba U, Sutton MA, Cape JN, Reis S, Sheppard LJ, Jenkins A, Grizzetti B, Galloway JN, Vitousek P, Leach A, Bouwman AF, Butterbach-Bahl K, Dentener F, Stevenson D, Amann M, Voss M. 2013. The global nitrogen cycle in the twenty-first century. Philos Trans R Soc Lond B Biol Sci 368:20130164. https://doi.org/10.1098/rstb.2013.0164.

2. Wheatley RM, Ford BL, Li L, Aroney STN, Knights HE, Ledermann R, East AK, Ramachandran VK, Poole PS. 2020. Lifestyle adaptations of Rhizobium from rhizosphere to symbiosis. Proc Natl Acad Sci U S A 117:23823-23834. https://doi.org/10.1073/pnas.2009094117.

3. Poole P, Ramachandran V, Terpolilli J. 2018. Rhizobia: from saprophytes to endosymbionts. Nat Rev Microbiol 16:291. https://doi.org/10.1038/nrmicro .2017.171.

4. MacLean AM, Finan TM, Sadowsky MJ. 2007. Genomes of the symbiotic nitrogen-fixing bacteria of legumes. Plant Physiol 144:615-622. https:// doi.org/10.1104/pp.107.101634.

5. Mauchline TH, Fowler JE, East AK, Sartor AL, Zaheer R, Hosie AHF, Poole PS, Finan TM. 2006. Mapping the Sinorhizobium meliloti 1021 solute-binding protein-dependent transportome. Proc Natl Acad Sci U S A 103: 17933-17938. https://doi.org/10.1073/pnas.0606673103.

6. Galardini M, Brilli M, Spini G, Rossi M, Roncaglia B, Bani A, Chiancianesi M, Moretto M, Engelen K, Bacci G, Pini F, Biondi EG, Bazzicalupo M, Mengoni A. 2015. Evolution of intra-specific regulatory networks in a multipartite bacterial genome. PLoS Comput Biol 11:e1004478. https://doi.org/10 .1371/journal.pcbi.1004478.

7. Lang C, Barnett MJ, Fisher RF, Smith LS, Diodati ME, Long SR. 2018. Most Sinorhizobium meliloti extracytoplasmic function sigma factors control accessory functions. mSphere 3:e00454-18. https://doi.org/10 .1128/mSphereDirect.00454-18.

8. Yurgel SN, Rice J, Mulder M, Kahn ML. 2010. GlnB/GInK PII proteins and regulation of the Sinorhizobium meliloti Rm1021 nitrogen stress response and symbiotic function. J Bacteriol 192:2473-2481. https://doi.org/10.1128/JB .01657-09.

9. Krol E, Blom J, Winnebald J, Berhörster A, Barnett MJ, Goesmann A, Baumbach J, Becker A. 2011. RhizoRegNet—a database of rhizobial transcription factors and regulatory networks. J Biotechnol 155:127-134. https://doi.org/10.1016/j.jbiotec.2010.11.004.

10. Bobrovskyy M, Vanderpool CK. 2013. Regulation of bacterial metabolism by small RNAs using diverse mechanisms. Annu Rev Genet 47:209-232. https://doi.org/10.1146/annurev-genet-111212-133445.

11. Schlüter JP, Reinkensmeier J, Barnett MJ, Lang C, Krol E, Giegerich R, Long SR, Becker A. 2013. Global mapping of transcription start sites and promoter motifs in the symbiotic alpha-proteobacterium Sinorhizobium meliloti 1021. BMC Genomics 14:156. https://doi.org/10.1186/1471-2164-14-156. 
12. Schlüter JP, Reinkensmeier J, Daschkey S, Evguenieva-Hackenberg $E$, Janssen S, Janicke S, Becker JD, Giegerich R, Becker A. 2010. A genomewide survey of sRNAs in the symbiotic nitrogen-fixing alpha-proteobacterium Sinorhizobium meliloti. BMC Genomics 11:245. https://doi.org/10 .1186/1471-2164-11-245.

13. Robledo M, García-Tomsig NI, Jiménez-Zurdo Jl. 2020. Riboregulation in nitrogen-fixing endosymbiotic bacteria. Microorganisms 8:384. https:// doi.org/10.3390/microorganisms8030384.

14. Robledo M, Peregrina A, Millán V, García-Tomsig NI, Torres-Quesada O, Mateos PF, Becker A, Jiménez-Zurdo Jl. 2017. A conserved $\alpha$-proteobacterial small RNA contributes to osmoadaptation and symbiotic efficiency of rhizobia on legume roots. Environ Microbiol 19:2661-2680. https://doi .org/10.1111/1462-2920.13757.

15. Torres-Quesada O, Millán V, Nisa-Martínez R, Bardou F, Crespi M, Toro N, Jiménez-Zurdo Jl. 2013. Independent activity of the homologous small regulatory RNAs AbcR1 and AbcR2 in the legume symbiont Sinorhizobium meliloti. PLoS One 8:e68147. https://doi.org/10.1371/journal.pone.0068147.

16. Baumgardt K, Šmídová K, Rahn H, Lochnit G, Robledo M, EvguenievaHackenberg E. 2016. The stress-related, rhizobial small RNA RcsR1 destabilizes the autoinducer synthase encoding mRNA $\sin /$ in Sinorhizobium meliloti. RNA Biol 13:486-499. https://doi.org/10.1080/15476286.2015.1110673.

17. Robledo M, Schlüter J-P, Loehr LO, Linne U, Albaum SP, Jiménez-Zurdo J, Becker A. 2018. An sRNA and cold shock protein homolog-based feedforward loop post-transcriptionally controls cell cycle master regulator CtrA. Front Microbiol 9:763. https://doi.org/10.3389/fmicb.2018.00763.

18. Robledo M, Frage B, Wright PR, Becker A. 2015. A stress-induced small RNA modulates alpha-rhizobial cell cycle progression. PLoS Genet 11: e1005153. https://doi.org/10.1371/journal.pgen.1005153.

19. Lagares A, Jr, Ceizel Borella G, Linne U, Becker A, Valverde C. 2017. Regulation of polyhydroxybutyrate accumulation in Sinorhizobium meliloti by the trans-encoded small RNA MmgR. J Bacteriol 199:e00776-16. https:// doi.org/10.1128/JB.00776-16.

20. Melior H, Li S, Madhugiri R, Stötzel M, Azarderakhsh S, Barth-Weber S, Baumgardt K, Ziebuhr J, Evguenieva-Hackenberg E. 2019. Transcription attenuation-derived small RNA rnTrpL regulates tryptophan biosynthesis gene expression in trans. Nucleic Acids Res 47:6396-6410. https://doi .org/10.1093/nar/gkz274.

21. Adams PP, Storz G. 2020. Prevalence of small base-pairing RNAs derived from diverse genomic loci. Biochim Biophys Acta Gene Regul Mech 1863: 194524. https://doi.org/10.1016/j.bbagrm.2020.194524.

22. Torres-Quesada O, Reinkensmeier J, Schlü̈er J-P, Robledo M, Peregrina A, Giegerich R, Toro N, Becker A, Jiménez-Zurdo Jl. 2014. Genome-wide profiling of $\mathrm{Hfq}$-binding RNAs uncovers extensive post-transcriptional rewiring of major stress response and symbiotic regulons in Sinorhizobium meliloti. RNA Biol 11:563-579. https://doi.org/10.4161/rna.28239.

23. del Val C, Rivas E, Torres-Quesada O, Toro N, Jiménez-Zurdo Jl. 2007. Identification of differentially expressed small non-coding RNAs in the legume endosymbiont Sinorhizobium meliloti by comparative genomics. Mol Microbiol 66:1080-1091. https://doi.org/10.1111/j.1365-2958.2007.05978.x.

24. del Val C, Romero-Zaliz R, Torres-Quesada O, Peregrina A, Toro N, Jiménez-Zurdo Jl. 2012. A survey of sRNA families in alpha-proteobacteria. RNA Biol 9:119-129. https://doi.org/10.4161/rna.18643.

25. Sheehan LM, Caswell CC. 2018. An account of evolutionary specialization: the AbcR small RNAs in the Rhizobiales. Mol Microbiol 107:24-33. https:// doi.org/10.1111/mmi.13869.

26. Caswell CC, Gaines JM, Ciborowski P, Smith D, Borchers CH, Roux CM, Sayood K, Dunman PM, Roop li RM. 2012. Identification of two small regulatory RNAs linked to virulence in Brucella abortus 2308. Mol Microbiol 85: 345-360. https://doi.org/10.1111/j.1365-2958.2012.08117.x.

27. Sheehan LM, Caswell CC. 2017. A 6-nucleotide regulatory motif within the AbcR small RNAs of Brucella abortus mediates host-pathogen interactions. mBio 8:e00473-17. https://doi.org/10.1128/mBio.00473-17.

28. Eisfeld J, Kraus A, Ronge C, Jagst M, Brandenburg VB, Narberhaus F. 2021. A LysR-type transcriptional regulator controls the expression of numerous small RNAs in Agrobacterium tumefaciens. Mol Microbiol 116: 126-139. https://doi.org/10.1111/mmi.14695.

29. Sheehan LM, Budnick JA, Blanchard C, Dunman PM, Caswell CC. 2015. A LysR-family transcriptional regulator required for virulence in Brucella abortus is highly conserved among the $\alpha$-proteobacteria. Mol Microbiol 98:318-328. https://doi.org/10.1111/mmi.13123.

30. Budnick JA, Sheehan LM, Ginder MJ, Failor KC, Perkowski JM, Pinto JF, Kohl KA, Kang L, Michalak P, Luo L, Heindl JE, Caswell CC. 2020. A central role for the transcriptional regulator VtIR in small RNA-mediated gene regulation in Agrobacterium tumefaciens. Sci Rep 10:14968. https://doi .org/10.1038/s41598-020-72117-0.

31. Overlöper A, Kraus A, Gurski R, Wright PR, Georg J, Hess WR, Narberhaus F. 2014. Two separate modules of the conserved regulatory RNA AbcR1 address multiple target mRNAs in and outside of the translation initiation region. RNA Biol 11:624-640. https://doi.org/10.4161/rna.29145.

32. Carrier MC, Laliberté G, Massé E. 2018. Identification of new bacterial small RNA targets using MS2 affinity purification coupled to RNA sequencing. Methods Mol Biol 1737:77-88. https://doi.org/10.1007/978-1 -4939-7634-8_5.

33. Lalaouna D, Prévost K, Eyraud A, Massé E. 2017. Identification of unknown RNA partners using MAPS. Methods 117:28-34. https://doi.org/10.1016/j .ymeth.2016.11.011.

34. Barnett MJ, Bittner AN, Toman CJ, Oke V, Long SR. 2012. Dual RpoH sigma factors and transcriptional plasticity in a symbiotic bacterium. J Bacteriol 194:4983-4994. https://doi.org/10.1128/JB.00449-12.

35. Robledo M, García-Tomsig NI, Matia-González AM, García-Rodríguez FM, Jiménez-Zurdo Jl. 2021. Synthetase of the methyl donor S-adenosylmethionine from nitrogen-fixing $\alpha$-rhizobia can bind functionally diverse RNA species. RNA Biol 18:1111-1123. https://doi.org/10.1080/15476286 .2020 .1829365 .

36. Robledo M, Matia-González AM, García-Tomsig NI, Jiménez-Zurdo ال 2018. Identification of small RNA-protein partners in plant symbiotic bacteria. Meth Mol Biol 1737:351-370. https://doi.org/10.1007/978-1-4939 -7634-8_20.

37. Becker A, Barnett MJ, Capela D, Dondrup M, Kamp PB, Krol E, Linke B, Ruberg S, Runte K, Schroeder BK, Weidner S, Yurgel SN, Batut J, Long SR, Puhler A, Goesmann A. 2009. A portal for rhizobial genomes: RhizoGATE integrates a Sinorhizobium meliloti genome annotation update with postgenome data. J Biotechnol 140:45-50. https://doi.org/10.1016/j.jbiotec .2008.11.006.

38. Lee HJ, Gottesman S. 2016. sRNA roles in regulating transcriptional regulators: Lrp and SoxS regulation by sRNAs. Nucleic Acids Res 44: 6907-6923. https://doi.org/10.1093/nar/gkw358.

39. diCenzo GC, Tesi M, Pfau T, Mengoni A, Fondi M. 2020. Genome-scale metabolic reconstruction of the symbiosis between a leguminous plant and a nitrogen-fixing bacterium. Nat Commun 11:2574. https://doi.org/ 10.1038/s41467-020-16484-2.

40. Beisel CL, Storz G. 2010. Base pairing small RNAs and their roles in global regulatory networks. FEMS Microbiol Rev 34:866-882. https://doi.org/10 .1111/j.1574-6976.2010.00241.x.

41. Richards GR, Vanderpool CK. 2011. Molecular call and response: the physiology of bacterial small RNAs. Biochim Biophys Acta Gene Regul Mech 1809:525-531. https://doi.org/10.1016/j.bbagrm.2011.07.013.

42. Tang G, Xing S, Wang S, Yu L, Li X, Staehelin C, Yang M, Luo L. 2017. Regulation of cysteine residues in LsrB proteins from Sinorhizobium meliloti under free-living and symbiotic oxidative stress. Environ Microbiol 19: 5130-5145. https://doi.org/10.1111/1462-2920.13992.

43. Sen A, Imlay JA. 2021. How microbes defend themselves from incoming hydrogen peroxide. Front Immunol 12:667343. https://doi.org/10.3389/ fimmu.2021.667343.

44. Tang G, Wang Y, Luo L. 2014. Transcriptional regulator LsrB of Sinorhizobium meliloti positively regulates the expression of genes involved in lipopolysaccharide biosynthesis. Appl Environ Microbiol 80:5265-5273. https://doi.org/10.1128/AEM.01393-14.

45. Lu D, Tang G, Wang D, Luo L. 2013. The Sinorhizobium meliloti LysR family transcriptional factor $L s r B$ is involved in regulation of glutathione biosynthesis. Acta Biochim Biophys Sin (Shanghai) 45:882-888. https://doi.org/ 10.1093/abbs/gmt083.

46. Mitsui H, Sato T, Sato Y, Ito N, Minamisawa K. 2004. Sinorhizobium meliloti $\mathrm{RpoH} 1$ is required for effective nitrogen-fixing symbiosis with alfalfa. Mol Genet Genomics 271:416-425. https://doi.org/10.1007/s00438-004-0992-x.

47. Luo L, Yao SY, Becker A, Ruberg S, Yu GQ, Zhu JB, Cheng HP. 2005. Two new Sinorhizobium meliloti LysR-type transcriptional regulators required for nodulation. J Bacteriol 187:4562-4572. https://doi.org/10.1128/JB.187 .13.4562-4572.2005.

48. Roux B, Rodde N, Jardinaud M-F, Timmers $T$, Sauviac L, Cottret L, Carrère S, Sallet E, Courcelle E, Moreau S, Debellé F, Capela D, de Carvalho-Niebel F, Gouzy J, Bruand C, Gamas P. 2014. An integrated analysis of plant and bacterial gene expression in symbiotic root nodules using laser-capture microdissection coupled to RNA sequencing. Plant J 77:817-837. https:// doi.org/10.1111/tpj.12442. 
49. Mika F, Hengge R. 2014. Small RNAs in the control of RpoS, CsgD, and biofilm architecture of Escherichia coli. RNA Biol 11:494-507. https://doi.org/ $10.4161 /$ rna.28867.

50. Vogel J, Wagner EG. 2007. Target identification of small noncoding RNAs in bacteria. Curr Opin Microbiol 10:262-270. https://doi.org/10.1016/j.mib 2007.06.001.

51. Georg J, Lalaouna D, Hou S, Lott SC, Caldelari I, Marzi S, Hess WR, Romby P. 2020. The power of cooperation: experimental and computational approaches in the functional characterization of bacterial sRNAs. Mol Microbiol 113:603-612. https://doi.org/10.1111/mmi.14420.

52. Wright PR, Georg J, Mann M, Sorescu DA, Richter AS, Lott S, Kleinkauf R, Hess WR, Backofen R. 2014. CopraRNA and IntaRNA: predicting small RNA targets, networks and interaction domains. Nucleic Acids Res 42: W119-W123. https://doi.org/10.1093/nar/gku359.

53. Carrier MC, Lalaouna D, Massé E. 2016. A game of tag: MAPS catches up on RNA interactomes. RNA Biol 13:473-476. https://doi.org/10.1080/15476286 .2016.1156830

54. Sharma CM, Papenfort K, Pernitzsch SR, Mollenkopf H-J, Hinton JCD, Vogel J. 2011. Pervasive post-transcriptional control of genes involved in amino acid metabolism by the Hfq-dependent GcvB small RNA. Mol Microbiol 81: 1144-1165. https://doi.org/10.1111/j.1365-2958.2011.07751.x.

55. Melamed S, Peer A, Faigenbaum-Romm R, Gatt YE, Reiss N, Bar A, Altuvia Y, Argaman L, Margalit H. 2016. Global mapping of small RNA-target interactions in bacteria. Mol Cell 63:884-897. https://doi.org/10.1016/j.molcel .2016.07.026.

56. Torres-Quesada O, Oruezabal RII, Peregrina A, Jofré E, Lloret J, Rivilla R, Toro N, Jiménez-Zurdo Jl, Jofre E, Lloret J, Rivilla R, Toro N, Jimenez-Zurdo JI. 2010. The Sinorhizobium meliloti RNA chaperone Hfq influences central carbon metabolism and the symbiotic interaction with alfalfa. BMC Microbiol 10:71. https://doi.org/10.1186/1471-2180-10-71.

57. Jiménez-Zurdo Jl, Robledo M. 2017. RNA silencing in plant symbiotic bacteria: insights from a protein-centric view. RNA Biol 14:1672-1677. https://doi.org/10.1080/15476286.2017.1356565.

58. Ceizel Borella G, Lagares A, Jr, Valverde C. 2018. Expression of the small regulatory RNA gene $m m g R$ is regulated negatively by AniA and positively by NtrC in Sinorhizobium meliloti 2011. Microbiology 164:88-98. https://doi.org/10.1099/mic.0.000586.

59. Povolo S, Casella S. 2000. A critical role for aniA in energy-carbon flux and symbiotic nitrogen fixation in Sinorhizobium meliloti. Arch Microbiol 174: 42-49. https://doi.org/10.1007/s002030000171.

60. Eberlein C, Baumgarten T, Starke S, Heipieper HJ. 2018. Immediate response mechanisms of Gram-negative solvent-tolerant bacteria to cope with environmental stress: cis-trans isomerization of unsaturated fatty acids and outer membrane vesicle secretion. Appl Microbiol Biotechnol 102:2583-2593. https://doi.org/10.1007/s00253-018-8832-9.

61. Delhaye A, Collet JF, Laloux G. 2019. A fly on the wall: how stress response systems can sense and respond to damage to peptidoglycan. Front Cell Infect Microbiol 9:380. https://doi.org/10.3389/fcimb.2019.00380.

62. Brenes-Álvarez M, Vioque A, Muro-Pastor AM. 2020. The integrity of the cell wall and its remodeling during heterocyst differentiation are regulated by phylogenetically conserved small RNA Yfr1 in Nostoc sp. strain PCC 7120. mBio 11:e02599-19. https://doi.org/10.1128/mBio.02599-19.

63. Muro-Pastor AM, Hess WR. 2020. Regulatory RNA at the crossroads of carbon and nitrogen metabolism in photosynthetic cyanobacteria. Biochim Biophys Acta Gene Regul Mech 1863:194477. https://doi.org/10.1016/j bbagrm.2019.194477.

64. Ruiz B, Le Scornet A, Sauviac L, Rémy A, Bruand C, Meilhoc E. 2019. The nitrate assimilatory pathway in Sinorhizobium meliloti: contribution to NO production. Front Microbiol 10:1526. https://doi.org/10.3389/fmicb.2019 .01526.

65. Patriarca EJ, Tatè R, laccarino M. 2002. Key role of bacterial $\mathrm{NH}_{4}{ }^{+}$metabolism in Rhizobium-plant symbiosis. Microbiol Mol Biol Rev 66:203-222. https://doi.org/10.1128/MMBR.66.2.203-222.2002.

66. Prell J, Bourdes A, Kumar S, Lodwig E, Hosie A, Kinghorn S, White J, Poole P. 2010. Role of symbiotic auxotrophy in the Rhizobium-legume symbioses. PLoS One 5:e13933. https://doi.org/10.1371/journal.pone.0013933.

67. Udvardi M, Poole PS. 2013. Transport and metabolism in legume-rhizobia symbioses. Annu Rev Plant Biol 64:781-805. https://doi.org/10.1146/ annurev-arplant-050312-120235.

68. Tkacz A, Bestion E, Bo Z, Hortala M, Poole PS. 2020. Influence of plant fraction, soil, and plant species on microbiota: a multikingdom comparison. mBio 11:e02785-19. https://doi.org/10.1128/mBio.02785-19.

69. Fagorzi C, Bacci G, Huang R, Cangioli L, Checcucci A, Fini M, Perrin E, Natali C, DiCenzo GC, Mengoni A. 2021. Nonadditive transcriptomic signatures of genotype-by-genotype interactions during the initiation of plant-Rhizobium symbiosis. mSystems 6:e00974-20. https://doi.org/10 .1128/mSystems.00974-20.

70. Gosai J, Anandhan S, Bhattacharjee A, Archana G. 2020. Elucidation of quorum sensing components and their role in regulation of symbiotically important traits in Ensifer nodulating pigeon pea. Microbiol Res 231: 126354. https://doi.org/10.1016/j.micres.2019.126354.

71. Bossi L, Figueroa-Bossi N. 2016. Competing endogenous RNAs: a targetcentric view of small RNA regulation in bacteria. Nat Rev Microbiol 14: 775-784. https://doi.org/10.1038/nrmicro.2016.129.

72. Miyakoshi M, Chao Y, Vogel J. 2015. Cross talk between ABC transporter mRNAs via a target mRNA-derived sponge of the GcvB small RNA. EMBO J 34:1478-1492. https://doi.org/10.15252/embj.201490546.

73. Denham EL. 2020. The sponge RNAs of bacteria-how to find them and their role in regulating the post-transcriptional network. Biochim Biophys Acta Gene Regul Mech 1863:194565. https://doi.org/10.1016/j.bbagrm .2020 .194565 .

74. Beringer JE. 1974. R factor transfer in Rhizobium leguminosarum. J Gen Microbiol 84:188-198. https://doi.org/10.1099/00221287-84-1-188.

75. Robertsen BK, Aman P, Darvill AG, McNeil M, Albersheim P. 1981. Hostsymbiont interactions : $\mathrm{V}$. The structure of acidic extracellular polysaccharides secreted by Rhizobium leguminosarum and Rhizobium trifolii. Plant Physiol 67:389-400. https://doi.org/10.1104/pp.67.3.389.

76. Cabanes D, Boistard P, Batut J. 2000. Symbiotic induction of pyruvate dehydrogenase genes from Sinorhizobium meliloti. Mol Plant Microbe Interact 13:483-493. https://doi.org/10.1094/MPMI.2000.13.5.483.

77. Schafer A, Tauch A, Jager W, Kalinowski J, Thierbach G, Puhler A. 1994. Small mobilizable multi-purpose cloning vectors derived from the Escherichia coli plasmids pK18 and pK19: selection of defined deletions in the chromosome of Corynebacterium glutamicum. Gene 145:69-73. https:// doi.org/10.1016/0378-1119(94)90324-7.

78. Simon R, Priefer U, Puhler A. 1983. A broad host range mobilization system for in vivo genetic engineering: transposon mutagenesis in gram negative bacteria. Nat Biotechnol 1:784-791. https://doi.org/10.1038/ nbt1183-784.

79. Bahlawane C, Mclntosh M, Krol E, Becker A. 2008. Sinorhizobium meliloti regulator MucR couples exopolysaccharide synthesis and motility. Mol Plant Microbe Interact 21:1498-1509. https://doi.org/10.1094/MPMI-21 $-11-1498$.

80. Khan SR, Gaines J, Roop RM, Farrand SK. 2008. Broad-host-range expression vectors with tightly regulated promoters and their use to examine the influence of TraR and TraM expression on Ti plasmid quorum sensing. Appl Environ Microbiol 74:5053-5062. https://doi.org/10.1128/AEM.01098-08.

81. Wei Z, Zhang W, Fang H, Li Y, Wang X. 2018. EsATAC: an easy-to-use systematic pipeline for ATAC-seq data analysis. Bioinformatics 34:2664-2665. https://doi.org/10.1093/bioinformatics/bty141.

82. Liao Y, Smyth GK, Shi W. 2019. The R package Rsubread is easier, faster, cheaper and better for alignment and quantification of RNA sequencing reads. Nucleic Acids Res 47:e47. https://doi.org/10.1093/nar/gkz114.

83. Robinson MD, Oshlack A. 2010. A scaling normalization method for differential expression analysis of RNA-seq data. Genome Biol 11:R25. https:// doi.org/10.1186/gb-2010-11-3-r25.

84. Robinson JT, Thorvaldsdóttir H, Winckler W, Guttman M, Lander ES, Getz G, Mesirov JP. 2011. Integrative genomics viewer. Nat Biotechnol 29:24-26. https://doi.org/10.1038/nbt.1754.

85. Pérez-Mendoza D, Felipe A, Ferreiro MD, Sanjuán J, Gallegos MT. 2019. AmrZ and FleQ co-regulate cellulose production in Pseudomonas syringae pv. tomato DC3000. Front Microbiol 10:746. https://doi.org/10.3389/ fmicb.2019.00746.

86. Becker A, Bergés $H$, Krol E, Bruand C, Rüberg $S, D C$, Lauber $E$, Meilhoc $E$, Ampe $F$, de Bruijn F, Fourment J, Francez-Charlot A, Kahn D, Küster $H$, Liebe C, Pühler A, Weidner S, Batut J. 2004. Global changes in gene expression in Sinorhizobium meliloti 1021 under microoxic and symbiotic conditions. Mol Plant Microbe Interact 17:292-303. https://doi.org/ 10.1094/MPMI.2004.17.3.292.

87. Olivares J, Casadesus J, Bedmar EJ. 1980. Method for testing degree of infectivity of Rhizobium meliloti strains. Appl Environ Microbiol 39: 967-970. https://doi.org/10.1128/aem.39.5.967-970.1980.

88. Larkin MA, Blackshields G, Brown NP, Chenna R, McGettigan PA, McWilliam $H$, Valentin F, Wallace IM, Wilm A, Lopez R, Thompson JD, Gibson TJ, Higgins DG. 2007. Clustal $W$ and Clustal $X$ version 2.0. Bioinformatics 23: 2947-2948. https://doi.org/10.1093/bioinformatics/btm404. 
89. Bailey TL, Boden M, Buske FA, Frith M, Grant CE, Clementi L, Ren J, Li WW, Noble WS. 2009. MEME Suite: tools for motif discovery and searching. Nucleic Acids Res 37:W202-W208. https://doi.org/10.1093/nar/gkp335.

90. Mann M, Wright PR, Backofen R. 2017. IntaRNA 2.0: enhanced and customizable prediction of RNA-RNA interactions. Nucleic Acids Res 45 W435-W439. https://doi.org/10.1093/nar/gkx279.

91. Keating SM, Bornstein BJ, Finney A, Hucka M. 2006. SBMLToolbox: an SBML toolbox for MATLAB users. Bioinformatics 22:1275-1277. https://doi.org/10 .1093/bioinformatics/btl111.

92. Bornstein BJ, Keating SM, Jouraku A, Hucka M. 2008. LibSBML: an API library for SBML. Bioinformatics 24:880-881. https://doi.org/10.1093/ bioinformatics/btn051.

93. Heirendt L, Arreckx S, Pfau T, Mendoza SN, Richelle A, Heinken A, Haraldsdóttir HS, Wachowiak J, Keating SM, Vlasov V, Magnusdóttir S, Ng CY, Preciat G, Žagare A, Chan SHJ, Aurich MK, Clancy CM, Modamio J, Sauls JT, Noronha A, Bordbar A, Cousins B, El Assal DC, Valcarcel LV, Apaolaza I, Ghaderi S, Ahookhosh M, Ben Guebila M, Kostromins A, Sompairac N, Le HM, Ma D, Sun Y, Wang L, Yurkovich JT, Oliveira MAP, Vuong PT, El Assal LP, Kuperstein I,
Zinovyev A, Hinton HS, Bryant WA, Aragón Artacho FJ, Planes FJ, Stalidzans E, Maass A, Vempala S, Hucka M, Saunders MA, Maranas CD, et al. 2019. Creation and analysis of biochemical constraint-based models using the COBRA Toolbox v.3.0. Nat Protoc 14:639-702. https://doi.org/10.1038/s41596-018-0098-2.

94. DiCenzo GC, Checcucci A, Bazzicalupo M, Mengoni A, Viti C, Dziewit L, Finan TM, Galardini M, Fondi M. 2016. Metabolic modelling reveals the specialization of secondary replicons for niche adaptation in Sinorhizobium meliloti. Nat Commun 7:12219. https://doi.org/10.1038/ncomms12219.

95. Vallenet D, Calteau A, Cruveiller S, Gachet M, Lajus A, Josso A, Mercier J, Renaux A, Rollin J, Rouy Z, Roche D, Scarpelli C, Medigue C. 2017. MicroScope in 2017: an expanding and evolving integrated resource for community expertise of microbial genomes. Nucleic Acids Res 45:D517-D528. https://doi.org/10.1093/nar/gkw1101.

96. Nelson M, Guhlin J, Epstein B, Tiffin P, Sadowsky MJ. 2018. The complete replicons of 16 Ensifer meliloti strains offer insights into intra- and interreplicon gene transfer, transposon-associated loci, and repeat elements. Microb Genom 4:e000174. https://doi.org/10.1099/mgen.0.000174. 